\title{
Detailed experimental results for high-trapping efficiency and narrow energy spread in a laser-driven accelerator
}

\author{
W. D. Kimura, * L. P. Campbell, C. E. Dilley, S. C. Gottschalk, and D. C. Quimby \\ STI Optronics, Inc., Bellevue, Washington 98004, USA \\ M. Babzien, I. Ben-Zvi, J. C. Gallardo, K. P. Kusche, I.V. Pogorelsky, J. Skaritka, and V. Yakimenko \\ Brookhaven National Laboratory, Upton, New York 11973, USA \\ D. B. Cline and F. Zhou \\ University of California, Los Angeles, Los Angeles, California 90095, USA \\ L. C. Steinhauer \\ University of Washington, Redmond Plasma Physics Laboratory, Redmond, Washington 98052, USA \\ R. H. Pantell \\ Stanford University, Stanford, California 94305, USA \\ (Received 12 April 2004; published 27 September 2004)
}

\begin{abstract}
Presented are details of the staged electron laser acceleration (STELLA) experiment, which demonstrated high-trapping efficiency and narrow energy spread in a staged laser-driven accelerator. Trapping efficiencies of up to $80 \%$ and energy spreads down to $0.36 \%(1 \sigma)$ were demonstrated. The experiment validated an approach that may be suitable for the basic design of a laser-driven accelerator system. In this approach, a laser-driven modulator together with a chicane creates a train of microbunches spaced apart by the laser wavelength. These microbunches are sent into a second laser-driven accelerator designed to efficiently trap the microbunches in the ponderomotive potential well of the laser electric field while maintaining a narrow energy spread. The STELLA scientific apparatus and procedures are described in detail. In-depth comparisons between the data and model are given including the predicted energy spectrum, energy-phase plot, and microbunch length profile. Data and model comparisons as a function of the phase delay between the microbunches and the accelerating wave are presented. The model is exercised to reveal how the high-trapping efficiency process evolves during the acceleration process.
\end{abstract}

DOI: 10.1103/PhysRevSTAB.7.091301

PACS numbers: 41.75.Jv, 41.60.Cr

\section{INTRODUCTION}

Laser acceleration, in which a laser beam either directly or indirectly accelerates electrons, continues to make great advances with acceleration gradients $>1 \mathrm{GV} / \mathrm{m}$ routinely demonstrated [1]. This technology may one day lead to more compact and less expensive accelerators as well as advanced accelerators for highenergy research.

A practical laser-driven accelerator (laser linac) requires certain characteristics. A large ensemble of electrons needs to be accelerated together while maintaining a narrow energy spread (monoenergetic). The former process, called trapping, is a familiar one in microwavedriven accelerators and entails causing the electrons to coalesce in the accelerating field phase space, such that the electrons are held within the ponderomotive potential well (bucket) of the accelerating field. The latter characteristic of being monoenergetic is important when staging the laser acceleration process, whereby the electrons

*Electronic address: wkimura@stioptronics.com are repeatedly accelerated in subsequent laser-driven acceleration devices. This is because it becomes difficult to capture and retain the electrons within the potential well bucket unless they occupy a narrow energy range. These effects will be shown in more detail later in this paper.

A number of experiments on laser acceleration research have been conducted at the Brookhaven National Laboratory (BNL) Accelerator Test Facility (ATF) [2], which features a microwave linac and a high-peak-power $\mathrm{CO}_{2}$ laser [3]. These experiments include pioneering work on inverse Cerenkov acceleration (ICA) [4] and inverse free electron lasers (IFEL) [5]. Combining these methods gave rise to the concept of using an IFEL to form microbunches for feeding into an ICA [6]. From this concept arose the staged electron laser acceleration (STELLA) experiment together with a change of focus to address system related issues, such as staging, rather than development of a specific acceleration mechanism. This change in goals freed the experiment from being tied to one specific acceleration scheme, e.g., ICA, and instead to select a scheme that provided the easiest means to demonstrate critical system capabilities. Thus, the STELLA 
experiment evolved into demonstrating, for the first time, staging between two IFELs [7]. One IFEL creates microbunches and the second IFEL accelerates them.

While this first demonstration [7] showed, for the first time, formation and acceleration of $\approx 3$ fs long microbunches in a laser-driven system, the quality of the trapping and energy spread of the accelerated electrons was not at a level needed for a practical laser linac as described above. Hence, improvements and modifications were made to the STELLA experiment and culminated in the first demonstration of high-trapping efficiency and monoenergetic acceleration [8]. Trapping efficiencies of up to $80 \%$ and energy spreads down to $0.36 \%(1 \sigma)$ were demonstrated.

The purpose of this paper is to bring to closure this experiment by presenting (i) complete details of the experimental system and methods, (ii) a complete set of the experimental results and corresponding model predictions, and (iii) model simulations to better understand the underlying processes occurring during the experiment.

Section II describes the experimental system and model. Section III shows the experimental results and their comparison with the model simulations. Additional modeling results are given in Sec. IV to elucidate the trapping and acceleration process. Conclusions are presented in Sec. V together with a brief description of new experiments underway to investigate laser wakefield acceleration (LWFA) [1].

\section{DESCRIPTION OF EXPERIMENT AND MODEL}

\section{A. Description of the overall approach}

As explained in Ref. [8], the STELLA approach starts with modulating the electron beam $(e$-beam) to create microbunches, which are then trapped and accelerated in a separate laser acceleration device. This basic approach can be applied to a wide range of different laser acceleration schemes. For convenience we chose to base our laser acceleration method on the IFEL [9] effect. In an IFEL the electrons travel through a periodic magnet array called a wiggler or undulator, as depicted in Fig. 1. This causes the electron trajectory to oscillate. A laser beam is also sent through the undulator collinear with the $e$-beam. Net energy exchange between the electrons and laser beam is possible if the resonance condition is satisfied [5],

$$
\gamma^{2}=\frac{\lambda_{w}}{2 \lambda_{L}}\left(1+\frac{K^{2}}{2}\right)
$$

where $\lambda_{L}$ is the laser wavelength, $\lambda_{w}$ is the undulator wavelength, $\gamma$ is the Lorentz factor, $K=e B_{o} \lambda_{w} / 2 \pi m c$, $e$ is the electron charge, $B_{o}$ is the peak magnetic field, $m$ is the electron rest mass, and $c$ is the speed of light. Higher energy exchange is possible if the wiggler or

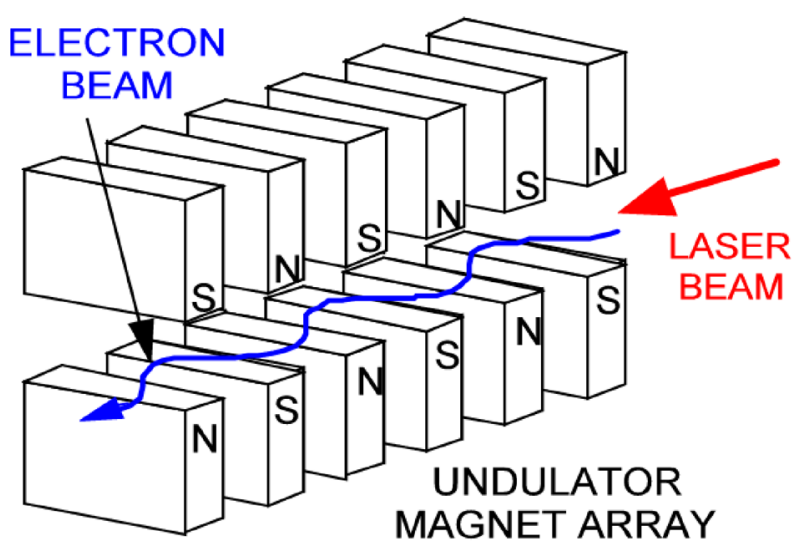

FIG. 1. (Color) Sketch of planar magnetic undulator array configured as an inverse free electron laser (IFEL). The $e$-beam and laser beam travel simultaneously and collinear through the gap of the undulator.

undulator is tapered, e.g., the gap or magnetic period becomes smaller along the magnet array [10].

IFELs are well-understood devices [10], and, as mentioned, an IFEL using an electromagnet (EM) undulator [5] was previously demonstrated at the ATF. As an additional factor adding to the convenience of using IFELs, STELLA was able to use permanent-magnet (PM) undulators [11] for the IFELs rather than pure EM-based ones. These PM devices were readily available from STI Optronics, Inc. [12], the lead institution for these experiments.

During the STELLA experiment, the ATF $\mathrm{CO}_{2}$ laser was capable of delivering multijoule laser pulses with a $180 \mathrm{ps}$ [full width at half maximum (FWHM)] pulse length. Subsequent to STELLA, the laser has been upgraded to deliver terawatt-level laser pulses. Table I lists the other parameters for the ATF $e$-beam and laser beam during the STELLA experiment.

Implementation of the STELLA approach consisted of first modulating the energy of the $e$-beam using an IFEL (called IFEL1) driven by the ATF $\mathrm{CO}_{2}$ laser beam. These modulated electrons are allowed to form microbunches by passing through a chicane located immediately downstream of IFEL1. These microbunches enter a second IFEL (called IFEL2) driven by the same ATF $\mathrm{CO}_{2}$ laser beam where they are trapped and accelerated.

Figure 2 shows a schematic layout of the experiment. The laser beam, seen entering from the top of the drawing, reflects off a convex $(f=-4 \mathrm{~m})$ copper mirror, which expands the laser beam before it passes through a $\mathrm{NaCl}$ lens $(f=50 \mathrm{~cm})$ and $\mathrm{NaCl}$ window into the electron beam line. This expansion is necessary to avoid damaging the window. (For clarity the laser beam is shown traveling in the plane of Fig. 2. In actuality, the laser beam enters the beam line perpendicular to the plane of the figure.) The laser beam passes through a 
TABLE I. Experimental parameters and values used in model.

\begin{tabular}{|c|c|c|}
\hline Parameter & Value & $\begin{array}{l}\text { Experimental value or } \\
\text { value used in model }\end{array}$ \\
\hline$e$-beam energy & $\approx 45 \mathrm{MeV}$ & Experiment and model \\
\hline$e$-beam intrinsic energy spread $(1 \sigma)$ & $0.03 \%$ & Experiment and model \\
\hline$e$-beam charge (total pulse) & $0.1 \mathrm{nC}$ & Experiment $^{\mathrm{a}}$ \\
\hline$e$-beam pulse length $(1 \sigma)$ & $\approx 3 \mathrm{ps}$ & Experiment $^{\mathrm{b}}$ \\
\hline$e$-beam normalized emittance $\left(\gamma \sigma \sigma^{\prime}\right)$ & $1.5 \mathrm{~mm} \mathrm{mrad}$ & Experiment and model \\
\hline$e$-beam $\beta$-function value at vertical waist & $0.7 \mathrm{~m}$ & Model $^{\mathrm{c}}$ \\
\hline$e$-beam $\beta$-function value at horizontal waist & $0.19 \mathrm{~m}$ & $M_{o d e l}{ }^{\mathrm{c}}$ \\
\hline Vertical $e$-beam waist location relative to center of IFEL2 & $2.1 \mathrm{~m}$ upstream & Model $^{\mathrm{c}}$ \\
\hline Horizontal $e$-beam waist location relative to center of IFEL2 & $0.7 \mathrm{~m}$ downstream & Model $^{\mathrm{c}}$ \\
\hline Laser pulse length (FWHM) & $\approx 180 \mathrm{ps}$ & Experiment and model \\
\hline Laser wavelength $\left(\mathrm{CO}_{2}\right.$ laser $)$ & $10.6 \mu \mathrm{m}$ & Experiment and model \\
\hline Laser polarization & Linear (vertical) & Experiment and model \\
\hline Estimated laser intensity inside IFEL1 & $8-9 \mathrm{GW} / \mathrm{cm}^{2}$ & Model $^{\mathrm{d}}$ \\
\hline Estimated laser intensity inside IFEL2 & $1.7-2.7 \mathrm{TW} / \mathrm{cm}^{2}$ & Experiment $^{\mathrm{e}}$ \\
\hline$e$-beam centroid offset from laser beam axis & $0.2 \mathrm{~mm}$ (vertical only) & Model $^{\mathrm{f}}$ \\
\hline
\end{tabular}

${ }^{a}$ Space-charge effects not activated in model because not significant (see text).

${ }^{\mathrm{b}} e$-beam pulse length much shorter than laser pulse length, therefore can ignore in model.

${ }^{c}$ Model values selected to match measured $e$-beam profiles at entrance and exit to IFEL2.

${ }^{\mathrm{d}}$ Intensity derived from model to yield same amount of measured modulation (see text).

${ }^{\mathrm{e}}$ Uncertainties in intensity due to diffraction effects inside IFEL2 (see text for intensities used in model).

${ }^{\mathrm{f}}$ Offset increased by $300 \mu \mathrm{m}$ in Fig. 13 (see text).

focus inside the beam line and then reflects off a $90^{\circ}$ parabolic mirror $(f=20 \mathrm{~cm})$ [13], which focuses the laser beam in the center of IFEL2. While traveling to IFEL2 the laser beam passes first through IFEL1 and the chicane situated between IFEL1 and IFEL2.

The $e$-beam enters from the right in Fig. 2, passes through a $1 \mathrm{~mm}$ diameter hole in the center of the parabolic mirror, and goes through a triplet whose purpose is to focus the $e$-beam in the center of IFEL2. Thus, the $e$-beam travels through IFEL1, the chicane, IFEL2, and finally enters an energy spectrometer at the end of the beam line, which analyzes the $e$-beam energy. These devices are described in more detail below.

\section{B. Electron beam control and characterization}

In the ATF linac, the electrons originate from a photoinjector whose photocathode is illuminated by a frequency-quadrupled Nd:YAG laser. Two $S$-band $(2856 \mathrm{MHz})$ linac sections are used to accelerate the electrons. Beam charge is measured at both the rf-gun exit and the end of the beam line utilizing Faraday cups.

A noteworthy characteristic of the $e$-beam is its exceptionally low normalized emittance $\left(\varepsilon_{n}<1.5 \mu \mathrm{m}\right)$ [14]. Emittance measurements are usually carried out at the high-energy transport line following the linac sections. In the transport line, there are three triplets and six beam profile monitors. Two methods are used to measure the

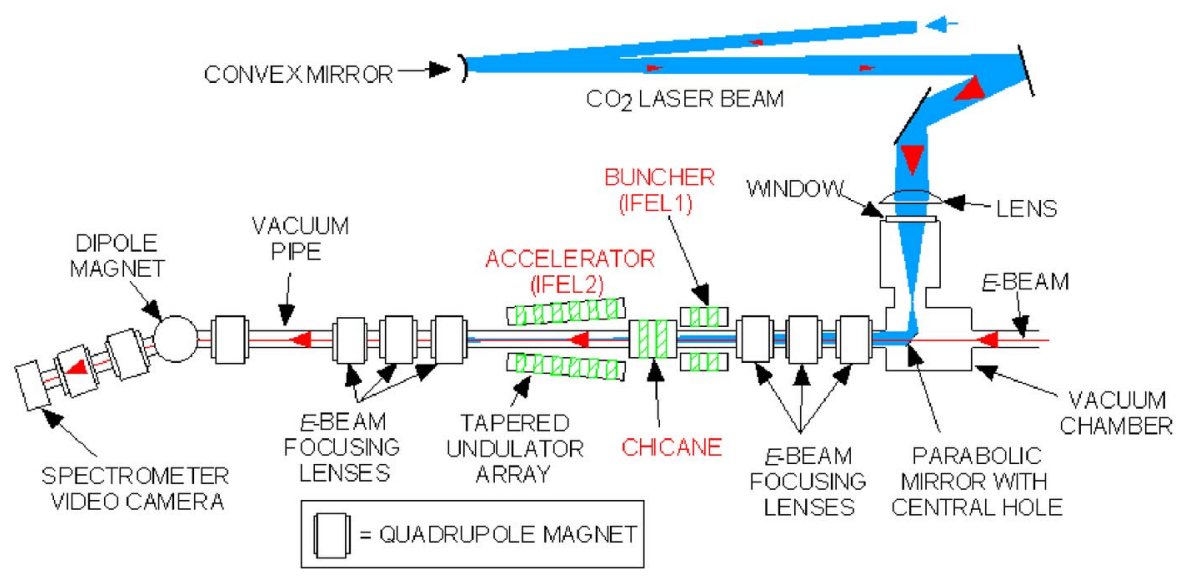

FIG. 2. (Color) Schematic layout for the STELLA experiment. 
emittance. The first is to use quad scanning [15] to measure beam sizes at one beam profile monitor. The second is to measure beam sizes using multiple monitors along the transport line [16]. Both techniques yield a normalized transverse emittance below $1.5 \mu \mathrm{m}$ during routine operations.

Following the transport line the electrons are bent horizontally by a $20^{\circ}$ dipole. The dipole acts as an energy spectrometer and can measure both $e$-beam energy and intrinsic energy spread. Intrinsic energy spread is measured in terms of horizontal beam size. This measurement indicates that the intrinsic energy spread is typically $<0.1 \%$ during routine operation $(\approx 0.03 \%$ during STELLA). Following the $20^{\circ}$ dipole, another $20^{\circ}$ dipole, oriented in the opposite direction, directs the $e$-beam into beam line \#1 where the STELLA experiment is located.

Two types of energy spectrometers are available at the end of beam line $\# 1$, featuring either $4^{\circ}$ or $90^{\circ}$ dipoles. The $90^{\circ}$ dipole spectrometer (not shown in Fig. 2) possesses high resolution (up to $10^{-4}$ ) to distinguish fine structures in the energy modulations, but the energy acceptance range is narrow $(< \pm 2 \%)$. The $4^{\circ}$-dipole energy spectrometer has a wide energy acceptance up to $\pm 20 \%$, but a lower measured resolution of $0.14 \%(1 \sigma)$. Another feature of the $4^{\circ}$ dipole is that its angle can be changed to $8^{\circ}$, which permits higher resolution, but correspondingly smaller energy acceptance.

The detectors for the spectrometers consist of phosphor screens viewed by CCD cameras. Peak emission of the phosphor is in the green at approximately $500 \mathrm{~nm}$, which matches well the peak response of the cameras. The cameras do not use image intensifiers; however, Peltier cooling is available to reduce noise. An estimated minimum charge of $\approx 10 \mathrm{pC}$ can be detected by the cameras. Note that this corresponds to $\approx 10 \%$ of the total charge delivered during the experiment. Therefore, features in the energy spectra with less than $10 \%$ of the total charge may have been barely visible or not detected.

During the experiment, the $e$-beam size is measured with beam position monitors (BPMs) located along the beam line and in between each of the major STELLA components. The model parameters (see Table I) for the $e$-beam were selected to yield similar $e$-beam dimensions during the transport of the $e$-beam within the model.

\section{Description of magnetic devices \\ 1. Undulator for IFEL1 (buncher)}

The undulator for IFEL1 is based upon the same hardware used to build the undulator for IFEL2 (see below) with several notable differences. The gap between the magnet arrays (see Fig. 1) is large $(4.1 \mathrm{~cm})$ to accommodate the laser beam. At the IFEL1 location the laser beam is over eight Rayleigh lengths upstream of its waist and consequently is over $1 \mathrm{~cm}$ in diameter. $\mathrm{NdFeB}$ permanent magnets are used in the magnet array; however, the large gap means the peak magnetic field in the center of the gap is relatively weak $(\approx 2 \mathrm{kG})$.

The relatively weak field together with a single laser beam driving both the IFELs meant the other parameters (wiggler period and number of periods) of the IFEL1 undulator had to be chosen so that the correct amount of energy modulation (i.e., $\pm 0.5 \%$ ) was imparted to achieve optimum microbunching at the entrance to IFEL2. Furthermore, to save costs it was desired to use a duplicate set of undulator hardware that was already available. This hardware had a magnet period of $3.3 \mathrm{~cm}$ (same as IFEL2); however, as we will explain next, it was possible to double the period to $6.6 \mathrm{~cm}$.

The laser beam traveling inside IFEL1 had reduced laser intensity due to its large size, but it also had diffraction rings, which further decreased the laser intensity in the center of the beam. Fortunately, if the IFEL1 undulator had five poles, then the combination of the $45 \mathrm{MeV} e$-beam, $2 \mathrm{kG}$ peak field, $6.6 \mathrm{~cm}$ magnet period, and lower laser intensity fortuitously resulted in close to the desired energy modulation. Hence, the other notable differences are IFEL1 had a magnet period of twice IFEL2 and only five poles rather than ten poles.

Figure 3 shows engineering drawings of the IFEL1 undulator. The longer magnet period is achieved by aligning adjacent magnets in each array in the same magnetic direction as shown in the figure. Iron poles are located between these magnet pairs. To achieve zero transverse offset of the $e$-beam traveling through the undulator, a single magnet is used between the poles at the entrance and exit. This effectively decreases the field strength at the entrance and end by half. Nonmagnetic "fake" aluminum poles and magnets are inserted in the empty slots thereby making it appear like a fully loaded magnet array. These fake poles and magnets helped maintain the proper alignment and spacing of the real poles and magnets. Steel field clamps located at the entrance and exit to the undulator are adjusted during magnetic measurements of the undulator to achieve near-zero steering of the $e$-beam.

Figure 4 is the measured on-axis magnetic field distribution of the IFEL1 undulator. Its short length means it has less than three full magnetic periods. End effects are significant and cause the "effective" magnetic period to be approximately $8 \mathrm{~cm}$ rather than the $6.6 \mathrm{~cm}$ physical period of the magnet spacing. The undulator $K$ parameter [see Eq. (1)] is approximately 1.5.

Since the laser beam polarization is vertical inside the beam line, the IFEL1 undulator gap is oriented vertical with respect to the beam line. This allows the undulator to be manually lowered and raised off the beam line pipe. This permits the device to be easily removed when other experiments need to use the same beam line. Kinematic feet (see Fig. 3) ensure the undulator alignment remains the same. 

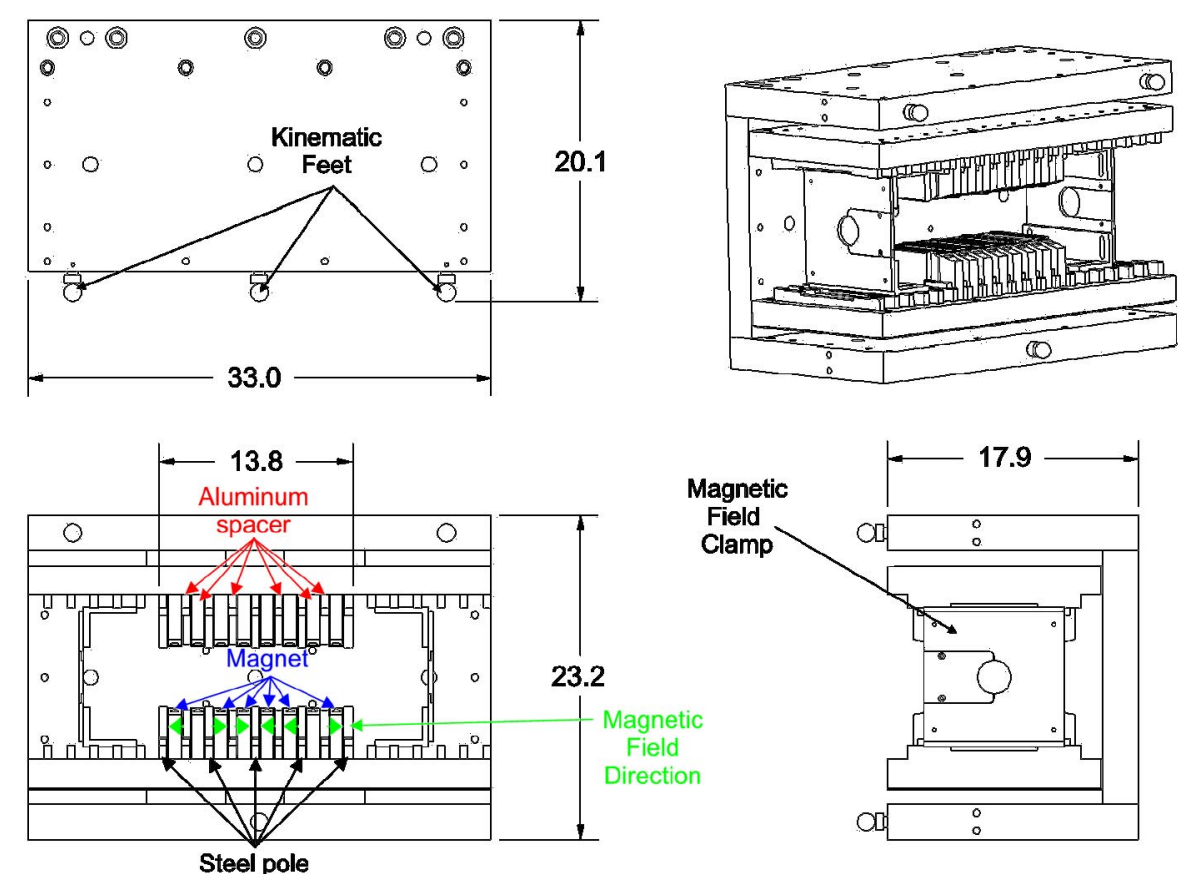

FIG. 3. (Color) Engineering drawings of IFEL1 undulator (buncher). All dimensions in centimeters.

As with any undulator there can be some focusing of the $e$-beam while interacting with the undulator field. Usually such focusing in these devices is not an issue since downstream $e$-beam optics can compensate for this effect. However, for this experiment such focusing could lead to bunch smearing in which electrons exiting the buncher follow slightly different path lengths to the accelerator (IFEL2). For example, electrons at the outer radius $r_{e}$ of the $e$-beam experience stronger focusing forces than electrons on axis resulting in the outer electrons leaving the buncher with a small, say, inwarddirected angle $\theta$. These outer electrons eventually intersect the on-axis electrons at the accelerator; however, they must take a longer path length. If this additional

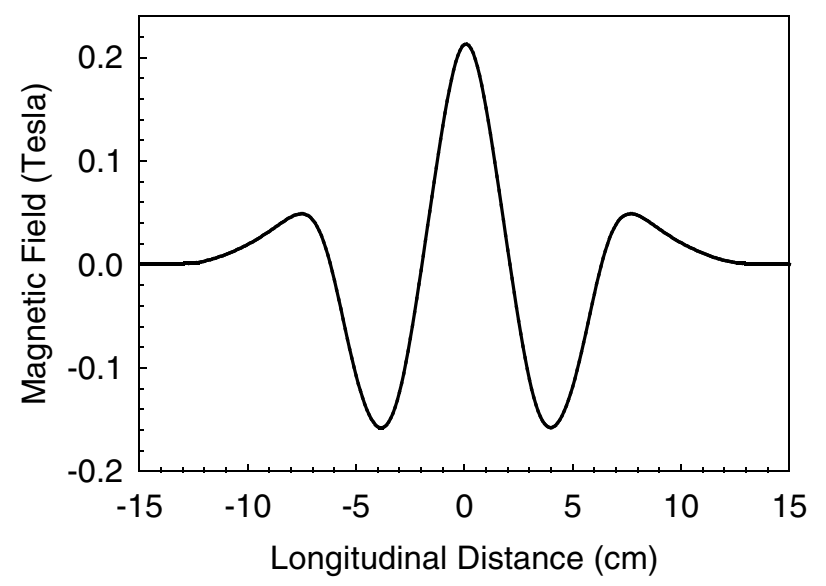

FIG. 4. Measured on-axis magnetic field distribution of IFEL1 undulator shown in Fig. 3. path length is comparable to the microbunch length of $1 \mu \mathrm{m}$, then bunch smearing will occur.

It is possible to estimate the degree to which this might be happening. Focusing by an undulator is typically characterized by the betatron wave number $k_{\beta}$ [17] given by

$$
k_{\beta}=\frac{\sqrt{2} \pi K}{\lambda_{w} \gamma}
$$

However, Eq. (2) assumes a uniform sinusoidal magnetic field distribution and, as seen in Fig. 4, this is clearly not the case for IFEL1. Equation (2) will tend to overestimate the betatron wave number and, hence, it represents an upper limit for the amount of focusing.

A more accurate, but somewhat laborious, method to calculate $k_{\beta}$ is to perform a magnetic code analysis of the field shown in Fig. 4 to derive all the magnetic force components acting on the $e$-beam. This process yields a value of $k_{\beta}=6.3 \times 10^{-3} \mathrm{~cm}^{-1}$ over an effective undulator length of $L=15 \mathrm{~cm}$. This is less than half the value given by Eq. (2).

If we next assume the $e$-beam entering the IFEL1 undulator has a radius of $r_{e}=0.5 \mathrm{~mm}$ and is perfectly collimated, then, due to the focusing force acting over a length $L$, an electron at $r_{e}=0.5 \mathrm{~mm}$ leaves the undulator with an angle

$$
\theta=r_{e} k_{\beta} \sin \left(k_{\beta} L\right)
$$

Substituting values into Eq. (3), we find for the buncher that $\theta=2.9 \times 10^{-5} \mathrm{rad}$.

The exit of IFEL1 is approximately $71 \mathrm{~cm}$ from the entrance to IFEL2. Thus, the additional path length $\Delta L$ 
traveled by the outer electron over this distance is only $3 \times 10^{-4} \mu \mathrm{m}$, which is much smaller than $1 \mu \mathrm{m}$, implying no bunch smearing caused by the undulator focusing.

\section{Chicane}

The chicane is a three-pole device with two NdFeB permanent magnets sandwiched between the poles. This creates a fixed magnetic field of alternating polarity between the three poles. The central iron pole also serves as a main electromagnet to provide a variable magnetic field superimposed on the static magnetic field. These fields are oriented perpendicular to the magnetic fields in IFEL1 and IFEL2 in order to minimize electron/laser interactions when the laser beam passes through the chicane with the $e$-beam. The end poles are also electromagnets and serve as trim coils to correct for steering of the $e$-beam whenever the main coil is energized. Figure 5 contains engineering drawings of the chicane showing these various features. Steel field clamps at the entrance and exit to the chicane ensure the chicane magnetic field does not interfere with and is not affected by other nearby components.

The measured magnetic field of the chicane is shown in Fig. 6. Large pole surface areas create flat regions of field. The fixed magnetic field forces the electrons to travel through a "V-shaped" trajectory [11] in which the faster electrons generated by IFEL1 traverse a shorter path than the slower ones, thereby causing the electrons to bunch together. By adjusting the insertion depth of the permanent magnets between the poles, the peak field value was

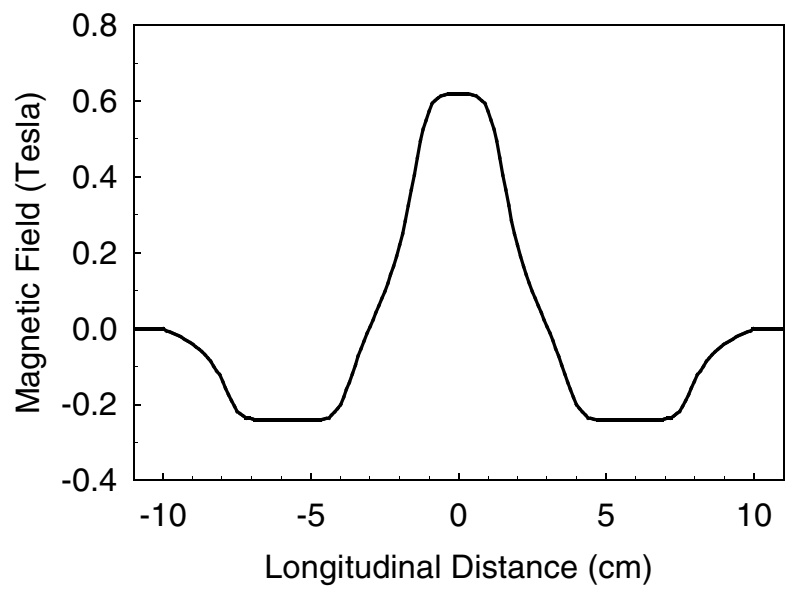

FIG. 6. Measured on-axis magnetic field distribution of chicane shown in Fig. 5.

chosen so that the electrons optimally bunch at IFEL2 when the energy modulation of the $45 \mathrm{MeV} e$-beam is $\pm 0.5 \%$. Since the peak transverse deflection distance of the electrons traveling along the V-shaped trajectory can be $>3 \mathrm{~mm}$, a rectangular-shaped vacuum pipe is used inside the chicane gap. Reference [11] contains more details of the chicane magnetic characteristics.

The chicane is even less like a conventional undulator than the IFEL1 undulator. Nonetheless, based upon Eq. (2), $k_{\beta}$ for the chicane is $\approx 3$ times higher than for the buncher. However, the distance from the chicane to IFEL 2 is only $1 / 3$ as much (i.e., $24 \mathrm{~cm}$ ). Hence, the $\Delta L$
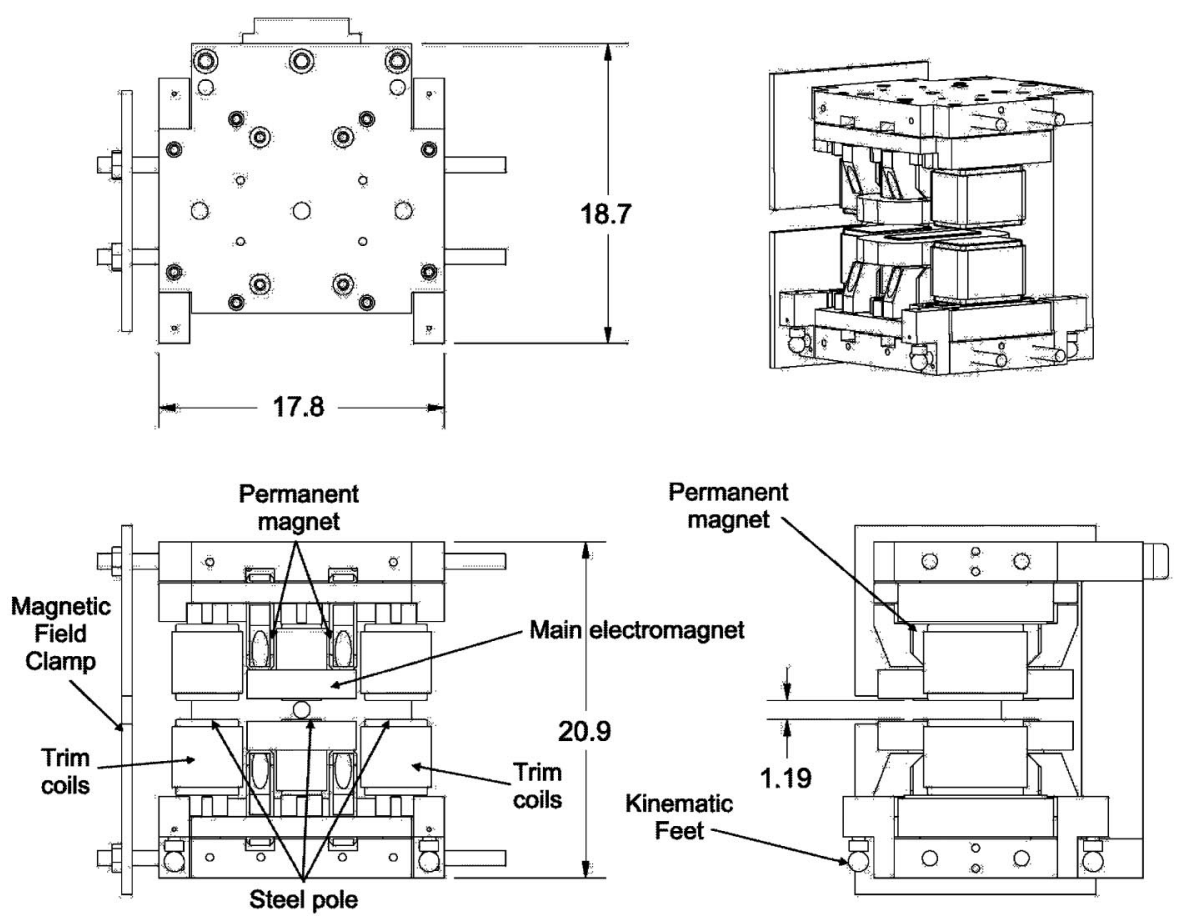

FIG. 5. Engineering drawings of chicane. All dimensions in centimeters. 
error for the chicane is still much less than $1 \mu \mathrm{m}$, implying once again no bunch smearing effects.

As explained in Ref. [8], since the $e$-beam pulse length is longer than the laser wavelength, a train of $\approx 85$ microbunches is created separated by $10.6 \mu \mathrm{m}$ with individual bunch lengths of $\approx 1-2 \mu \mathrm{m}$ (equivalent to $\approx$ 3-7 fs). Control of the phase delay between these microbunches and the laser field in IFEL2 is achieved by adjusting the main coil of the chicane. This field causes the electrons to travel a slightly different V-shaped trajectory, thereby, either lengthening or shortening the total travel distance through the chicane. This enables resynchronizing the microbunches with the accelerating portion of the laser field by changing the time when the electrons exit the chicane. Data as a function of the chicane phase delay will be shown later.

Usage of the chicane also makes the entire system more compact with a total length of $1.2 \mathrm{~m}$ from the entrance of IFEL1 to the egress of IFEL2. Since the magnetic field of the chicane is orthogonal to the IFEL1 and IFEL2 undulators, its gap is oriented horizontal. This permitted the chicane to be slid away from the beam line via rails with a counterweight on the opposite side of the beam line for stability.

\section{Undulator for IFEL2 (accelerator)}

The IFEL2 undulator consisted of NdFeB permanent magnets and iron poles arranged to give a magnetic period of $3.3 \mathrm{~cm}$ and a total length of $33 \mathrm{~cm}$. Partial strength magnets are used at the entrance and end to achieve zero transverse offset of the $e$-beam. Hence, the undulator had eight full-strength magnetic periods. When configured as a uniform gap undulator with a gap of $7.9 \mathrm{~mm}$, the wiggler $K$ parameter is 2.96. During STELLA, the gap is tapered in which the gap spacing is reduced at the exit by $11 \%$ (equivalent to $12 \%$ energy taper).

Tapering [18] is the key for achieving high-trapping efficiency and sufficient energy gain to separate the accelerated electrons from the background electrons. The laser field nominally accelerates the trapped electrons by the amount of energy taper of the undulator. The taper causes the resonance condition to continuously change along the undulator; therefore, a minimum amount of laser intensity is needed to ensure the electrons gain enough energy along the undulator to maintain the resonance condition. If the laser intensity falls below this minimum, then the electrons drop out of resonance along the undulator and gain little energy. In fact, the performance of a tapered undulator operating at laser intensities below threshold is in general worse than an untapered undulator operating at the same laser intensities.

Although the theoretical maximum amount of electrons in the microbunches produced by IFEL1 and the chicane is $\approx 50 \%$, with high enough laser intensity driving IFEL2 it is possible to trap much more than $50 \%$ of the electrons [19]. It is also possible to attain higher energy gain than the amount of energy taper by judicious selection of the incoming $e$-beam energy and the synchrotron period within IFEL2. This will be illustrated later.

Figures 7 and 8 are engineering drawings and the measured magnetic field distribution of the IFEL2 undulator, respectively. The slight increase in the magnetic field strength towards the exit end of the undulator can
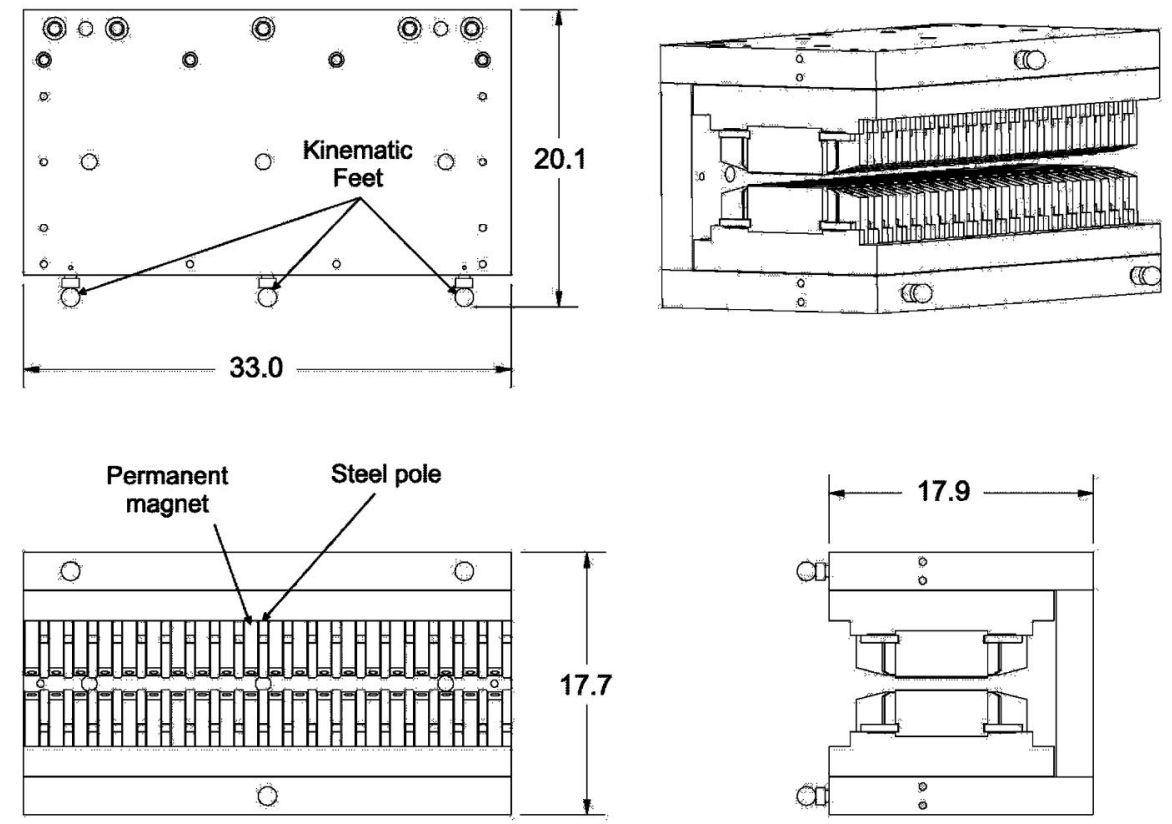

FIG. 7. Engineering drawings of IFEL2 undulator (accelerator). All dimensions in centimeters. 


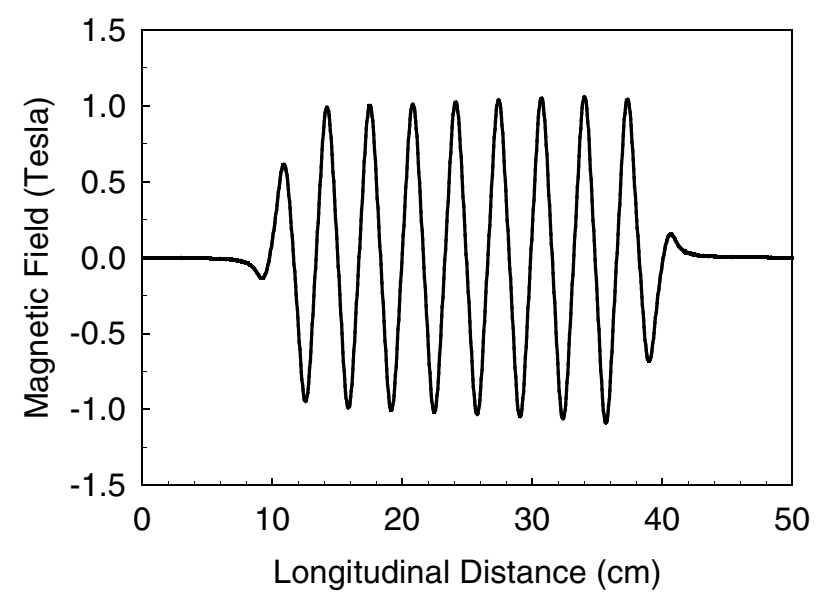

FIG. 8. Measured on-axis magnetic field distribution of IFEL2 undulator shown in Fig. 7.

be seen in Fig. 8. Field end clamps are not necessary for this device. The gap is also oriented vertical, which allowed this device to be manually raised or lowered onto the beam line. However, since the gap of the IFEL2 undulator is small with $<1 \mathrm{~mm}$ clearance between the tapered end and the beam line vacuum tube, a pair of manual lifting stages are used to lower or raise the undulator.

\section{Laser beam control and characterization}

\section{Measurement of laser beam characteristics}

A combination of standard and nonstandard techniques is utilized to characterize the ATF $\mathrm{CO}_{2}$ laser beam. The temporal shape of the 180 ps laser pulse length is too fast for conventional infrared detectors, but too long for an autocorrelator detector available at the ATF. One effective means for measuring the laser beam temporal pulse shape is to use $\mathrm{x}$-ray emission data taken during a Compton scattering experiment [20] in which the $e$-beam pulse is scanned in time along the laser pulse length. The results of this measurement are given in Fig. 9. This shows a socalled gain-switched spike at the leading edge that is typical of $\mathrm{CO}_{2}$ laser amplifier systems.

Standard calorimetric methods are used to measure the time-integrated laser beam pulse energy with a nominal $10 \%$ accuracy. A shot-to-shot variance of $\pm 12 \%$ was measured during the experiment. As explained later, the delivered laser pulse energy was intentionally chosen to be just above the minimum value necessary to drive the tapered undulator in order to achieve a narrow energy spread of the accelerated electrons. Consequently, the natural $\pm 12 \%$ pulse-to-pulse energy fluctuations meant that some shots had pulse energies insufficient to drive the tapered undulator and some had extra energy. The former case leads to poor trapping and acceleration; the latter leads to good trapping and acceleration, but slightly wider energy spreads.

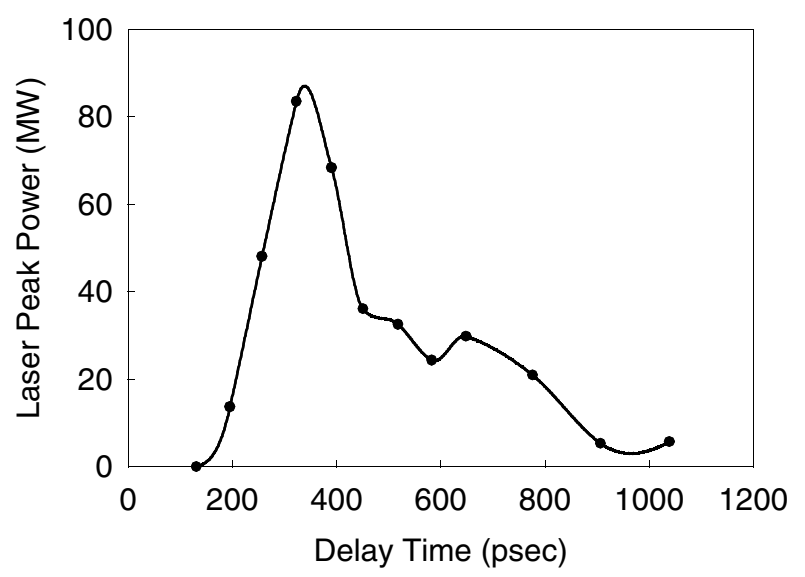

FIG. 9. ATF laser beam pulse shape obtained using x-ray emission data from a Compton scattering measurement [20].

Determination of the effective laser intensity in the center of IFEL2 is critical for making comparisons with the model. However, the terawatt intensity levels preclude direct measurement of this intensity. Therefore, the laser intensity must be determined through indirect means. Estimating the laser energy delivered requires accounting for optical losses as the laser beam travels through various optics along the STELLA laser beam transport line. The uncoated $\mathrm{NaCl}$ lens and beam line window (see Fig. 2) have Fresnel reflection losses of $\approx 4 \%$ per surface for a total of $16 \%$. The $1 \mathrm{~mm}$ hole in the parabolic mirror, which is in the center of the Gaussian-shaped laser beam, removes $\approx 13 \%$ of the laser pulse energy. IR camera images of the laser beam waist at the center of IFEL2 reveal an Airy pattern. This pattern is caused by various diffraction effects from the hole in the parabolic mirror and minor clipping of the outer edges of the laser beam due to limiting apertures along the laser beam transport line. The central lobe of the Airy pattern has a diameter of approximately $1.5 \mathrm{~mm}$ FWHM. Based upon prior experience, roughly $50 \%-80 \%$ of the laser energy is in the central lobe.

\section{Laser beam alignment}

The invisible, high-peak-power nature of the $\mathrm{CO}_{2}$ laser beam poses challenges when aligning the beam through the STELLA optical transport line. In particular, the laser beam focus at IFEL2 is highly sensitive to misalignment of the laser beam on the parabolic mirror. Unfortunately, the parabolic mirror is inside the beam line under vacuum, which prevents direct access to the mirror. Therefore, to aid in the alignment process, red $\mathrm{HeNe}$ lasers are used extensively to simulate both the $e$-beam and the $\mathrm{CO}_{2}$ laser beam.

A HeNe laser beam is sent down the center of the beam line pipe to represent the $e$-beam. Constricting apertures along the way are the $1 \mathrm{~mm}$ hole in the parabolic mirror and the $5 \mathrm{~mm}$ inner diameter of the beam line pipe 
passing through IFEL2. A second HeNe laser beam is introduced into the laser beam transport line and is aligned collinear with the $\mathrm{CO}_{2}$ laser beam. Since most of the optical elements along the transport line are reflective ones, the HeNe laser beam simulates well the $\mathrm{CO}_{2}$ laser beam trajectory. The exception is the $\mathrm{NaCl}$ lens where its focal length changes significantly at visible wavelengths and decentering errors on the lens can cause decentering errors on the parabolic mirror resulting in distortions of the laser focus at IFEL2.

Hence, once initial alignment of the optics is attained with the HeNe laser beams, the process must be repeated with the $\mathrm{CO}_{2}$ laser beam. Generally, this only requires fine-tuning the distance of separation between the $\mathrm{NaCl}$ lens and parabolic mirror, and the alignment of the parabolic mirror. The former controls the focus size of the laser beam at IFEL2. The latter affects distortion of the laser beam focus.

To visualize the laser beam focus, a mirror located in the BPM just upstream of the IFEL2 undulator directs the laser beam out of the beam line and into an IR camera located at the equivalent distance as the center of IFEL2. For this measurement the laser beam is greatly attenuated by operating without the $\mathrm{CO}_{2}$ amplifier and with attenuators situated along the transport line and in front of the camera.

The parabolic mirror is mounted on a remotecontrolled tilt stage that permits adjustment of its pitch and yaw. However, remote control of its transverse position is not available. This had to be achieved by adjustment of the optics outside the beam line pipe, e.g., translating the $\mathrm{NaCl}$ lens. This limitation in the adjustability of the parabolic mirror made it difficult to always achieve symmetric laser focus spots at IFEL2.

\section{Laser beam/e-beam timing synchronization}

The 3 ps $e$-beam pulse must be positioned at the desired time delay within the $180 \mathrm{ps}$ laser pulse. There are two steps in this process. The first is to obtain gross timing synchronization using a strip line current detector for the $e$-beam on beam line \#1 and a fast $\mathrm{HgCdTe}$ photodetector ( $\leq 1 \mathrm{~ns}$ rise time) located nearby the strip line detector. The outputs from these detectors are connected via a short coax cable to a nearby fast oscilloscope whose traces can be remotely monitored. This procedure allows synchronizing the $e$-beam and laser pulses to within $\approx 1 \mathrm{~ns}$.

Finer synchronization is achieved by adjusting an optical delay line that controls when $1.06 \mu \mathrm{m}$ laser light from the photocathode driver laser arrives at the $\mathrm{CO}_{2}$ laser system. This $1.06 \mu \mathrm{m}$ light is used to slice out the $180 \mathrm{ps} \mathrm{CO}_{2}$ laser pulse utilizing a semiconductor slicing technique [21]. Thus, varying when the $1.06 \mu \mathrm{m}$ light arrives changes when the $180 \mathrm{ps} \mathrm{CO}_{2}$ laser pulse is generated. The optical delay line has a delay range of $1.3 \mathrm{~ns}$ and subpicosecond step size permitting precision control of the delay time.

From previous experience, a convenient procedure for adjusting the optical delay line is to watch for signs of energy modulation on the spectrometer. For this procedure the IFEL1 undulator, IFEL2 undulator, and chicane are removed. An untapered undulator, essentially identical to the IFEL 2 undulator except for the lack of taper, is inserted where the IFEL2 undulator is normally located. Achieving energy modulation from the untapered undulator is relatively easy because unlike the tapered undulator it does not have a minimum laser threshold needed to drive the undulator.

In general, the $e$-beam and laser pulses are not temporally overlapped after the $1 \mathrm{~ns}$ gross synchronization. The optical delay is systematically scanned to both earlier and later times until modulation is detected. This delay is further scanned until maximum modulation is detected corresponding to the peak of the laser pulse (see Fig. 9). Then the delay time is adjusted to be slightly after this peak point (e.g., $\approx 400$ ps in Fig. 9). This position in time yields high-peak power while avoiding shot-to-shot fluctuations in the immediate vicinity of the peak. Jitter in this delay time is negligible compared to variations in the laser pulse-to-pulse energy and a slow drift in the spatial overlap of the $e$-beam and laser pulses within IFEL2 (see Sec. III).

\section{E. Model description}

The STELLA model, discussed in Ref. [22], was upgraded for these series of measurements. This model was originally developed for the precursor STELLA experiment [7] where staging between two IFELs was first demonstrated. The configurations and devices used in these two experiments were different enough to require modifying the code. Specifically, because the IFEL1 undulator and the chicane are short devices with only a few periods, end effects dominate the field distribution, which means the distribution is not purely sinusoidal. Thus the usual FEL equations, which assume a wiggleaveraged interaction between sinusoidal laser and wiggler fields of extended length, no longer provide reasonably accurate descriptions of the interaction strength in such a device. Hence the STELLA code was upgraded to employ a non-wiggle-averaged model. In addition, the code is able to use the measured 1D longitudinal magnetic field distributions for each of the undulators and the chicane, i.e., Figs. 4, 6, and 8.

The basic approach is to calculate the electron trajectory based on the magnetic field (neglecting perturbations induced by the laser) and then calculate the electron acceleration based on interaction with the laser. The plane-polarized laser electric field is expressed as

$$
\vec{E}_{L}=\hat{x} E_{o}(x, y, z) \cos \left[k_{L} z+\omega t+\phi(x, y, z)\right] .
$$


Here $z$ is the longitudinal coordinate (along the wiggler axis) and $(x, y)$ are the transverse coordinates (wiggle direction and $B$-field gradient direction, respectively). The phase of the optical beam $\phi(x, y, z)$ represents the deviation of the laser beam phase relative to an ideal forward-directed plane wave. The optical field wave number is $k_{L}=2 \pi / \lambda_{L}$ and frequency is $\omega=c k_{L}$. Higher harmonics of the optical field are neglected. The generalized magnetic field distribution is

$$
\vec{B}=\hat{y} B_{y}(z) .
$$

In this generalized nonsinusoidal case we neglect transverse $B$-field gradients and associated $e$-beam focusing. Using the Lorentz force equation,

$$
\frac{d}{d t}(\gamma m \vec{\nu})=\frac{e}{c}(\vec{\nu} \times \vec{B}),
$$

where $\vec{\nu}$ is the velocity vector for the electron, we can readily derive the trajectory of each electron

$$
\begin{gathered}
\beta_{x}(z)=\theta_{x}(z)=\frac{e}{m c^{2}} \int \frac{B_{y}(z) d z}{\gamma}, \\
x(z)=\frac{e}{m c^{2}} \iint \frac{B_{y}(z) d z^{2}}{\gamma} .
\end{gathered}
$$

The quantity $\theta_{x}$ is the transverse angle of the electron trajectory projected onto the $(x, z)$ plane. Using the relationship

$$
\gamma=\frac{1}{\sqrt{1-\beta_{x}^{2}-\beta_{y}^{2}-\beta_{z}^{2}}},
$$

and a small angle approximation, the axial velocity of each electron is

$$
\beta_{z} \cong 1-\frac{1}{2 \gamma^{2}}\left[1+\gamma^{2}\left(\theta_{x}^{2}+\theta_{y}^{2}\right)\right] .
$$

Here we also include the transverse angle of the electron trajectory $\theta_{y}$ projected onto the $(y, z)$ plane to represent the effects of finite $e$-beam emittance. The work done on the electron by the laser field is given by

$$
\frac{d}{d t}\left(\gamma m c^{2}\right)=-e E_{x} \nu_{x}
$$

where $\nu_{x}$ is the velocity component in the $x$ direction.

It is convenient to define a $(\gamma, \psi)$ "resonant window," which moves forward through space and time at a rate defined for an electron resonant with a particular hypothetical sinusoidal wiggler field,

$$
B_{y, \text { res }}(z)=B_{o, \text { res }}(z) \cos \int k_{w, \text { res }}\left(z^{\prime}\right) d z^{\prime},
$$

where $B_{o, \text { res }}(z)$ is the magnetic field amplitude and $k_{w, \text { res }}(z)=2 \pi / \lambda_{w, \text { res }}(z)$ is the wiggler wave number. Note that this hypothetical wiggler field does not neces- sarily exist; it is simply a theoretical construct used to define the phase $\psi$ and the associated resonant window used in the simulation. The rate of motion of the resonant window is defined to be equal to that of an electron resonant with the hypothetical wiggler field, i.e., $\beta_{z \text {,res }}=$ $1-\lambda_{L} / \lambda_{w, \text { res }}$. The dynamics of the electron energy and phase within the resonant window are calculated based on the actual magnetic field distribution given in Eq. (5). In general, the electron phase dynamics within the resonant window will deviate significantly from the near-resonant motion for those cases in which the magnetic field distribution is much different from the sinusoidal distribution defined in Eq. (12).

The most convenient definition for the electron phase $\psi$ is then

$$
\psi=\int_{0}^{z} k_{w, \text { res }}\left(z^{\prime}\right) d z^{\prime}+k_{s} z-\omega t .
$$

This allows the fast oscillating terms $k_{L} z-\omega t$ in Eq. (4) to be eliminated from the equations. Since the rate of motion of the resonant window is predetermined, the phase of the optical wave within the window is also known at every step of the simulation. Substituting into Eq. (11), we obtain

$$
\frac{d \gamma}{d z}=-\sqrt{2} e_{s} \theta_{x}(z) \cos \left[\psi+\phi-\int k_{w, \text { res }}\left(z^{\prime}\right) d z^{\prime}\right],
$$

where the relationship $d \gamma / d z \sim(1 / c)(d \gamma / d t)$, i.e., $v_{z}=$ $d z / d t \sim c$, has been utilized. This acceleration equation represents the instantaneous dot product of the electron transverse wiggle velocity, $\beta_{x}=\theta_{x}$, with the laser electric field distribution within the window. The laser beam electric field amplitude $E_{o}$ is represented by the normalized rms quantity $e_{s}(x, y, z)=e E_{o}(x, y, z) / \sqrt{2} m c^{2}$. The phase of each electron within the resonant window is given by

$$
\begin{aligned}
\frac{d \psi}{d z} & =k_{w, \text { res }}-k_{L}\left(1-\beta_{z}\right) \\
& =k_{w, \text { res }}-\frac{k_{L}}{2 \gamma^{2}}\left[1+\gamma^{2}\left(\theta_{x}^{2}+\theta_{y}^{2}\right)\right] .
\end{aligned}
$$

Equations (14) and (15), together with the trajectory Eqs. (7) and (8), form the basic generalized FEL simulation model without wiggle averaging. These equations can be compared to the more standard wiggle-averaged equations

$$
\begin{gathered}
\frac{d \gamma}{d z}=\frac{-G e_{s} K \sin (\psi+\phi)}{\sqrt{2} \gamma}, \\
\frac{d \psi}{d z}=k_{w}-\frac{k_{L}}{2 \gamma^{2}}\left[1+\frac{K^{2}}{2}+\gamma^{2}\left(\theta_{x}^{2}+\theta_{y}^{2}\right)\right],
\end{gathered}
$$

where a sinusoidal magnetic field of the form given in Eq. (12) is assumed to exist. The FEL interaction term on the right side of Eq. (16) includes the planar-wiggler 
coupling factor

$$
G=J_{0}(A)-J_{1}(A) ; \quad A=\frac{K^{2}}{4+2 K^{2}},
$$

which accounts for the effect of axial flutter of the electron velocity. The non-wiggle-averaged model was tested by comparison to the more standard wiggle-averaged model, i.e., Eqs. (16) and (17). It was found that the non-wiggle-averaged equations give identical results for the case of an integral number of periods of sinusoidal wiggler field. (The effect of axial flutter, which is included directly in the non-wiggle-averaged model, averages to zero over an integral number of wiggler periods.)

Although the model has the capability to approximate the effects of space charge, separate analysis and modeling work [23] indicates that space charge and coherent synchrotron radiation are not significant for the STELLA experimental conditions. Hence, this capability was not utilized during the model comparisons in this paper.

In the model results shown here, 5000 electrons are initially distributed uniformly over the laser beam phase and sent individually through the entire STELLA system from the entrance of IFEL1 to the spectrometer. The measured resolution of the spectrometer $[0.14 \%(1 \sigma)]$ is incorporated in the presentation of the final energy spectrum to permit direct comparison of the model predictions with the data. As shown later, the electrons in the microbunches can be color coded to facilitate following their evolution through energy-phase space.

The model assumes ideal Gaussian beam focusing of the laser beam and used the effective Rayleigh range for the actual optics described earlier. However, edge diffraction effects (i.e., Fresnel rings) were also present during the experiment. These effects were more pronounced at the IFEL1 location than at the IFEL2 position. This made it more difficult to quantify the laser intensity at IFEL1. Nevertheless, the amount of energy modulation at IFEL1 is a well-known function of the laser intensity. Hence, it is possible to use the model to estimate the effective laser intensity at IFEL1.

The model is able to simulate partial spatial overlap and angular error of the $e$-beam and laser beam within IFEL2, which, as will be shown later, is important for understanding the data results.

\section{EXPERIMENTAL RESULTS AND DISCUSSION}

\section{A. Data reduction process}

The output from the spectrometer consists of a video image where energy dispersion is in the horizontal direction (e.g., see video images given in Ref. [22]) along the raster lines of the camera. The $e$-beam focus through the spectrometer is adjusted so that the beam is spread out in the vertical direction, thereby providing redundant dispersion information along each raster line. This made it possible to greatly reduce noise in the data by averaging together 20 successive raster lines to yield an energy spectrum.

The area under each data spectrum is then normalized to the 5000 electrons in the corresponding model simulation. Hence, although the $e$-beam charge might vary from shot to shot resulting in fainter or brighter video images, all the data spectra are normalized to 5000 counts. This procedure allowed comparing the different $e$-beam shots with one another and the model. (This is valid because space-charge affects are negligible [23].) One caveat, however, is that the fainter spectra corresponding to relatively low $e$-beam charge may have contained features below the detection limit of the camera. This might lead to disagreements with the model, which would show these features.

It should be noted that data spectra obtained during the experiment, including all those presented in this paper, did not appear to display any dependence with respect to the amount of beam charge; that is, the same amount of energy gain and spread was observed for the same laser power even though the $e$-beam charge decreased or increased. This is consistent with space charge having no affect on the microbunching and trapping process. An example is shown later in Fig. 14.

\section{B. $\boldsymbol{e}$-beam spectrum without laser beam present}

We first show the $e$-beam-only spectrum in Fig. 10 along with the model prediction. The abscissa is the energy shift of the $e$-beam from its initial value. In Fig. 10(a) the abscissa is plotted on the same scale as the other spectra shown later. The ordinate represents the number of electrons where, as mentioned, the area under the data curve has been normalized to represent the equivalent 5000 particles of the model. Figure 10(b) is an enlarged view to show more details of the spectrum. Since the intrinsic energy width of the $e$-beam is only $0.03 \%$, the width of the spectrum is limited by the resolution of the spectrometer. We see there is good agreement between the data (red curve) and model (histogram); in particular, the peak values agree well indicating the area normalization factor is correct.

\section{C. $e$-beam spectrum after modulation by IFEL1}

Figure 11(a) shows the $e$-beam spectrum data after modulation by IFEL1 and transport through the chicane. IFEL2 was removed for this shot and, hence, this gives the condition of the $e$-beam before entering IFEL2. Since the $e$-beam pulse length ( $\approx 3 \mathrm{ps}$ ) is much shorter than the laser pulse length $(\approx 180 \mathrm{ps})$, the laser intensity can be considered constant over the duration of the $e$-beam pulse. However, since the laser wavelength $(10.6 \mu \mathrm{m})$ is much shorter than the $e$-beam pulse length, different electrons interact with different phases of the optical field resulting in a uniform energy modulation of the $e$-beam 

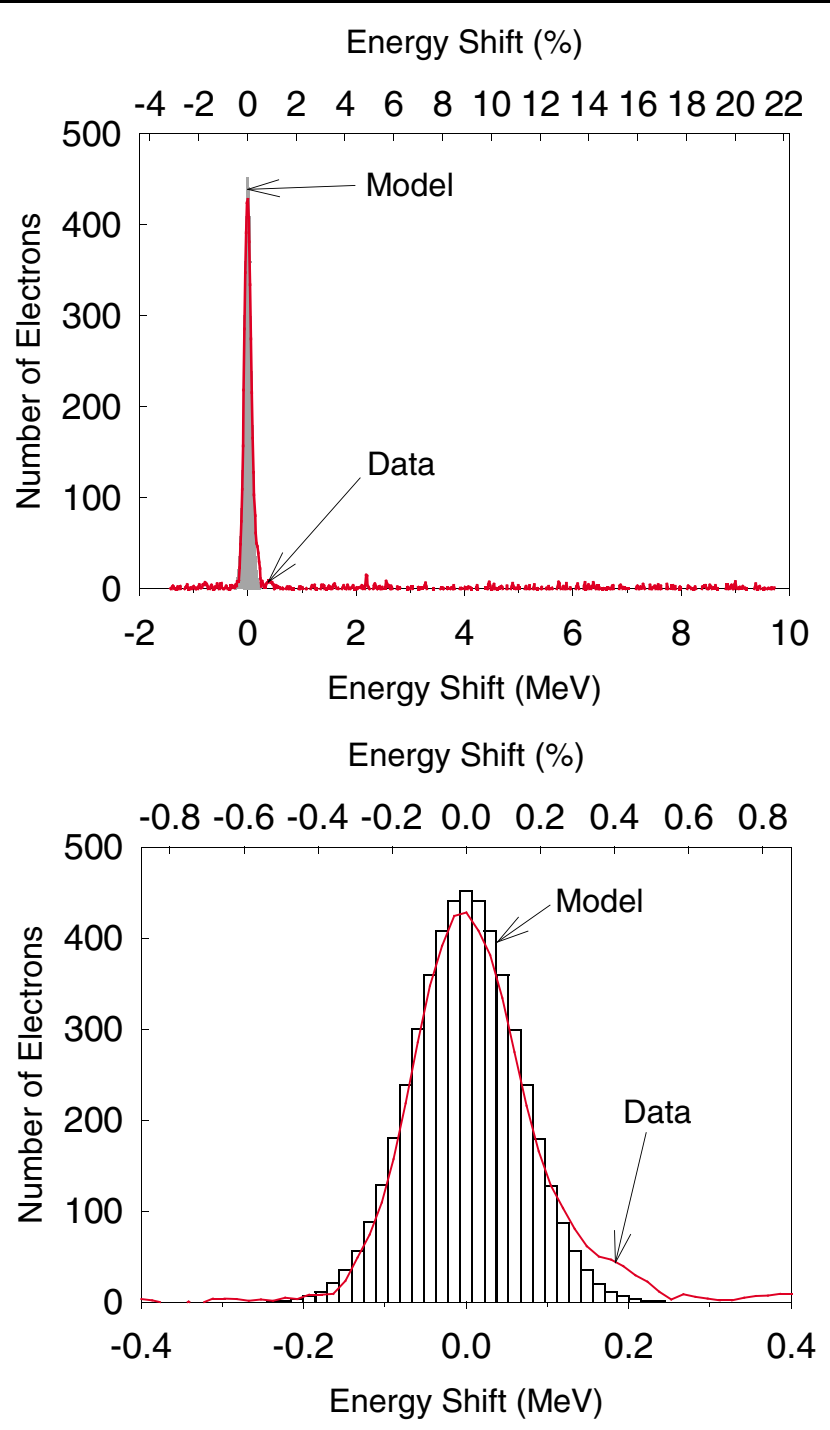

FIG. 10. (Color) Comparison of typical $e$-beam-only energy spectrum and model prediction after normalizing the areas of the data with the model. (a) Energy spectrum with data as solid (red) line and model as histogram. (b) Expanded view of spectrum shown in (a).

[8]. This causes the energy spectrum to display a doublepeaked profile centered at zero energy shift relative to the mean $e$-beam energy ( $45 \mathrm{MeV})$.

Overlaid on the data curve in Fig. 11(a) is the model prediction in which the laser intensity at IFEL1 in the model is set to $8.7 \mathrm{GW} / \mathrm{cm}^{2}$. The good agreement between the data and the model implies that the effective laser intensity within IFEL1 during the experiment was close to $8-9 \mathrm{GW} / \mathrm{cm}^{2}$. Both curves display an approximate $\pm 0.5 \%$ energy modulation, which is the desired target value.

Figure 11 also depicts other results from the model that are very useful for interpreting the energy spectrum. Figure 11(b) is a projection of the energy distribution onto phase space where $2 \pi$ of phase corresponds to one period of the laser wavelength. Since this phase-space diagram represents the $e$-beam after the chicane, the electrons have bunched together in phase. The result is the formation of a microbunch, as can be seen in Fig. 11(c), which is a projection of the electrons in phase onto the equivalent longitudinal spatial coordinate. Figure 11(c) indicates the microbunch length is $<2 \mu \mathrm{m}$ (equivalent to $<7 \mathrm{fs}$ ). Because the center portion of the $\mathrm{S}$ shaped distribution of electrons in Fig. 11(b) is not exactly horizontal, this implies the electrons are actually slightly overbunched, resulting in an elongated microbunch length.

As mentioned earlier, a train of roughly 85 microbunches is formed with each shot. These microbunches are separated from each other by $10.6 \mu \mathrm{m}$ with a background level of electrons in between, as seen in Fig. 11(c). The model simulates only one of these microbunches under the assumption that the other microbunches have similar characteristics. The energy spectrometer integrates over all the microbunches as well as the background electrons; whereas, the model energy histogram represents only the electrons within one optical period.

For simple sinusoidal energy modulation, a maximum of $\approx 50 \%$ of the electrons within the $e$-beam pulse can form into a microbunch. The rest are distributed uniformly over phase. To help keep track of these 50\% electrons within the microbunch, they are labeled as red in Fig. 11(b). As we will show later, during the hightrapping-efficiency process, these microbunch electrons as well as neighboring electrons in phase are captured and accelerated as a group.

One final note: The model histogram in Fig. 11(a) is wider than the maximum energy spread seen in Fig. 11(b) because the model includes spreading of the energy spectrum to simulate the resolution limit of the spectrometer. However, the model does not apply this spreading effect when generating the energy-phase plot.

\section{High-trapping efficiency results}

Figure 12 shows an example of high-trapping efficiency after sending the microbunches through IFEL2. Figure 12(a) compares the data spectrum with the model, Fig. 12(b) is the corresponding energy-phase diagram produced by the model, and Fig. 12(c) is the model prediction for the longitudinal density distribution. The laser intensity at IFEL2 used in the model is $1.7 \mathrm{TW} / \mathrm{cm}^{2}$, which is within the range of the estimated intensity during the experiment (see Table I). In Fig. 12(a), the data shows a large fraction of electrons accelerated by over $7 \mathrm{MeV}$ ( $>15 \%$ energy gain) with a peak acceleration of over $9 \mathrm{MeV}$ (20\% energy gain). A simple integration of the data curve indicates that $\approx 80 \%$ of the electrons are within the accelerated peak. This peak has an energy spread of $1.2 \%(1 \sigma)$.

The model prediction in Fig. 12(a) also indicates a large number of accelerated electrons. Figure 12(b) shows 


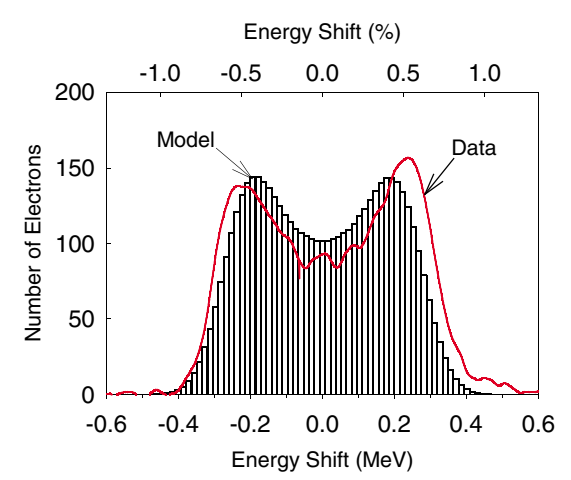

(a)

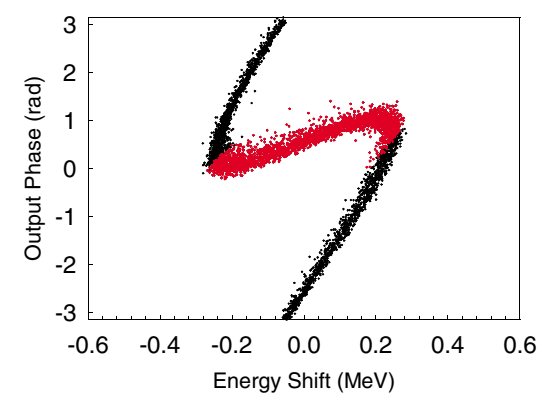

(b)

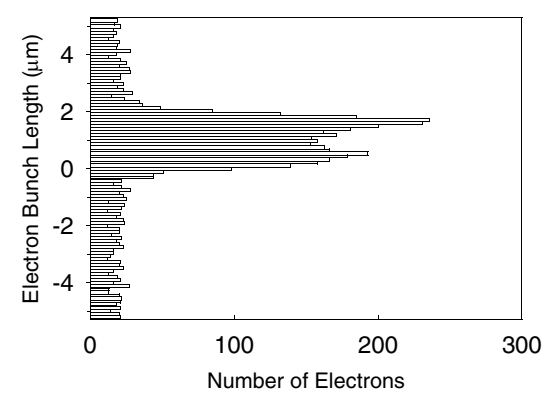

(c)

FIG. 11. (Color) Typical data energy spectrum and model prediction for $e$-beam after modulation by IFEL1 and traveling through chicane (IFEL2 is off). (a) Electron energy spectrum for data (solid red curve) and model (histogram). (b) Energy-phase plot for model prediction shown in (a). The initial microbunch electrons are colored in red. (c) Electron longitudinal density distribution derived from (b).

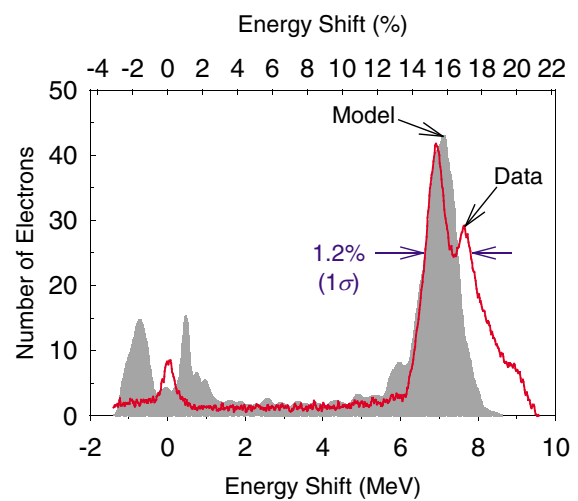

(a)

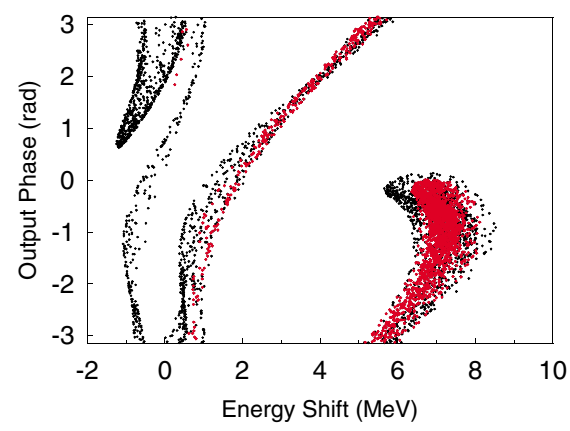

(b)

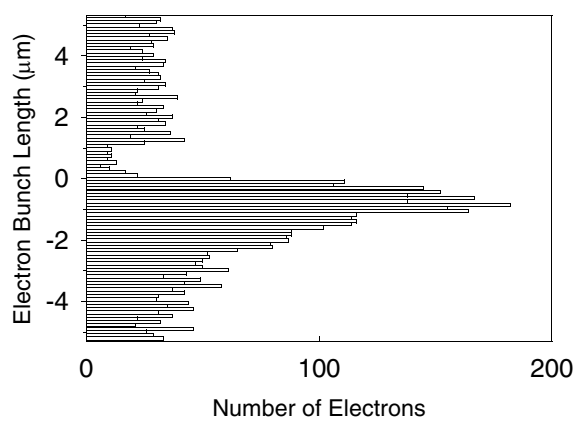

(c)

FIG. 12. (Color) Example of data and model prediction showing high-trapping efficiency. (a) Electron energy spectrum for data (solid red curve) and model (histogram). (b) Energy-phase plot for model prediction shown in (a). The initial microbunch electrons shown in red in Fig. 11(b) are again indicated in red. (c) Electron longitudinal density distribution derived from (b). 
that these trapped electrons include most of the original microbunch electrons as well as a significant portion of other electrons. Using the model, we will later show how these neighboring electrons become trapped with the microbunch electrons as the electrons travel down the tapered undulator. Only a small number of the original microbunch electrons are untrapped, as seen by the red dots distributed along the middle portion of the plot.

As can be seen in Fig. 12(b), the trapped and accelerated electrons have become well separated in energyphase space from the untrapped electrons. Within the ponderomotive bucket of the accelerating field, the trapped electrons have just begun to rotate counterclockwise in phase space, i.e., they have just begun to execute one synchrotron oscillation.

Part of the aim of this experiment was to demonstrate monoenergetic laser acceleration. Hence, the experiment was designed to limit the amount of synchrotron oscillation since a significant amount of phase rotation of the trapped electrons within the bucket leads to broadening of their energy spread. As will be shown below, the minimum energy spread occurs when the trapped electrons barely start one synchrotron oscillation.

The untrapped electrons are distributed in a rather complicated pattern over the remainder of phase space and consist of essentially three groups of electrons. Two of these groups stretch vertically across $2 \pi$ of phase and the third "V-shaped" group extends almost $\pi$ in phase downward from the top of the plot. In reality, this Vshaped group "attaches" to the bottom of the plot where the other two groups intersect the abscissa. Similarly, the trapped electrons attach to the top of the plot where the rightmost group of untrapped electrons intersects the top of the plot. (The evolution of these complicated phase pathways is further revealed later in Fig. 15.) Thus, during their travel through the tapered undulator, the entire ensemble of electrons becomes distributed over nearly $4 \pi$ of phase. How these three groups of untrapped electrons are distributed within phase space affects the energy spectrum profile, which integrates over all phase. This accounts for the pair of peaks seen near zero energy shift in Fig. 12(a).

The data display only a single peak at zero energy shift. It is not clear why there is this difference, which was consistently observed during the experiment. Attempts were made to adjust certain model parameters, such as the magnetic field strength of the undulators, which is sensitive to changes in the room temperature [24]. But, this did not improve the agreement. As noted in Ref. [24], the lack of exact values for certain experimental parameters, such as the precise trajectory of the $e$-beam through the threedimensional magnetic field, does limit the ability of the model to predict all the fine details seen in the data. Indeed, the model only used a 1D measurement of the magnetic field for all the devices.
The model indicates in Fig. 12(c) that the microbunch length has not broadened significantly during the trapping process. Although the data has a wider energy spread for the trapped electrons than the model, it is still possible the microbunch length during the experiment was similar to the model prediction. In any case, since the data demonstrated higher trapping efficiency than the model, this implies the number of electrons in the microbunch during the experiment appears to be greater than what the model indicates in Fig. 12(c).

\section{E. Narrow energy spread results}

Figure 13(a) shows the results for the case of narrow energy spread of the trapped electrons. The data now displays a narrow peak $[0.36 \%(1 \sigma)]$ of accelerated electrons at approximately $7 \mathrm{MeV}$ ( $\approx 16 \%$ energy gain). Roughly $14 \%$ of the electrons are within this narrow peak; the rest are distributed over a wide energy range. [The two dips labeled fiducial lines in Fig. 13(a) are caused by $0.08 \mathrm{~mm}$ thick wires attached to the phosphor screen at the output of the spectrometer. Although these wires were also present in the data for Fig. 12(a) and the other spectra shown in this paper, they are not always noticeable because they may lie in regions where there is little signal.]

One major difference between Figs. 12 and 13 is the laser intensity in IFEL2 is lower at $\approx 1.3 \mathrm{TW} / \mathrm{cm}^{2}$ in the model. This intensity corresponds to the minimum needed to drive the tapered undulator. At this threshold laser intensity, the electrons in the microbunch have barely begun one synchrotron oscillation, as can be seen in Fig. 13(b). This results in the narrow energy spread seen in the model prediction.

Another noteworthy difference between Figs. 12 and 13 is the large number of electrons at intermediate energies in Fig. 13, which results in significantly lower trapping efficiency. The model provides clues for a possible reason for these partially accelerated electrons. As mentioned earlier, the model simulates the actual $e$-beam focus within IFEL2, which during the experiment was slightly asymmetric with respect to the center of the undulator. At the entrance to the undulator, the $e$-beam was mostly circular in shape with a horizontal width of $0.53 \mathrm{~mm}$ FWHM $(1 \sigma=0.23 \mathrm{~mm})$. At the exit, the horizontal width of the $e$-beam was $0.21 \mathrm{~mm} \mathrm{FWHM} \mathrm{(} 1 \sigma=$ $0.09 \mathrm{~mm}$ ) and the vertical size of the $e$-beam was about a factor of 2-3 larger. The $\beta$-function values and $e$-beam waist locations listed in Table I were chosen to achieve a similar asymmetric focusing in the model.

If the laser beam is centered over the $e$-beam inside the undulator, i.e., there is little mismatch of spatial overlap between the two beams, then this asymmetric $e$-beam focus has only minor effects on the trapping efficiency, as demonstrated in Fig. 12. However, if there is enough transverse offset between the beams, then the asymmetric 


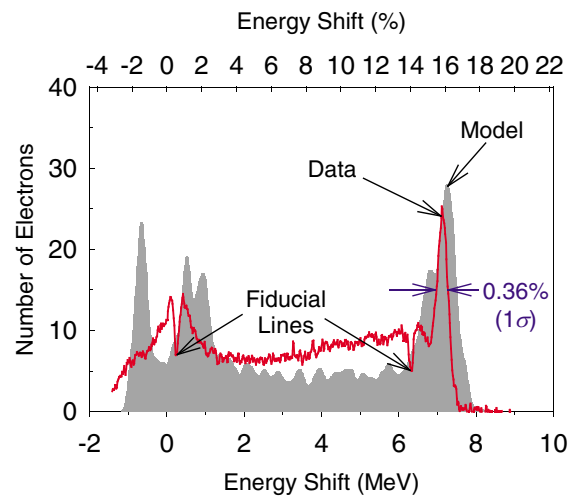

(a)

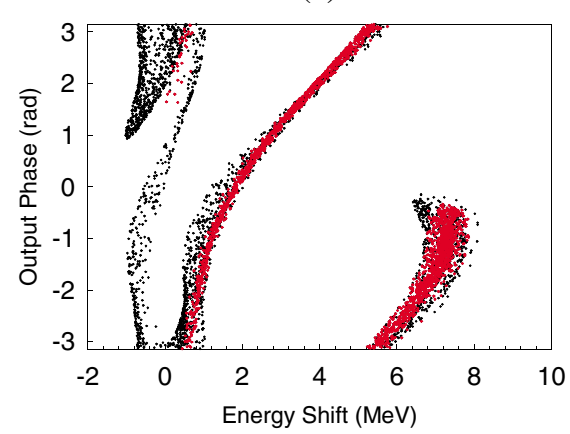

(b)

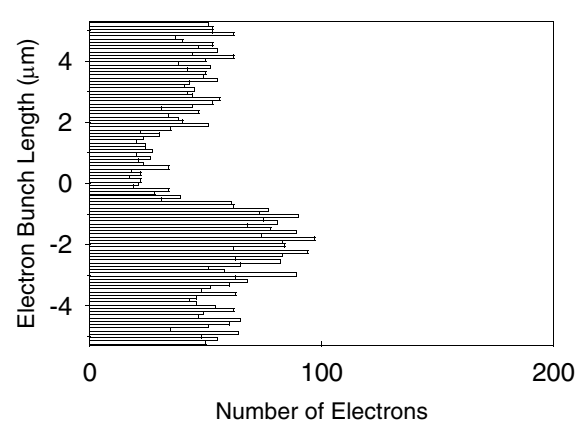

(c)

FIG. 13. (Color) Example of data and model prediction showing narrow energy spread. (a) Electron energy spectrum for data (solid red curve) and model (histogram). (b) Energy-phase plot for model prediction shown in (a). The initial microbunch electrons shown in red in Fig. 11(b) are again indicated in red. (c) Electron longitudinal density distribution derived from (b).

$e$-beam focus permits a portion of the electrons to initially interact with the laser beam at the beginning of the undulator and then leave the laser beam prematurely along the undulator and/or enter a region of reduced laser intensity. This would result in some of the electrons receiving only partial acceleration.

The model appears to support this hypothesis. If all the model parameters used in Fig. 12 are kept the same except for the lower laser peak power, then the model predicts high-trapping efficiency with narrow energy spread. In other words, the model does not predict a large number of electrons at intermediate energies (see Ref. [24]). However, if the transverse spatial offset between the laser beam and $e$-beam inside IFEL2 is increased by $300 \mu \mathrm{m}$, then the model predicts the results shown in Fig. 13(a). During the experiment, a slow drift of the spatial overlap between the $e$-beam and laser beam was observed; therefore, a $300 \mu \mathrm{m}$ offset is quite plausible.

With this offset, the model in Fig. 13(b) clearly shows a large fraction of the initial electrons in the microbunch are not trapped and are spread over a wide energy range (see streak of red dots in center of plot). Not surprising, this partial acceleration process also tends to smear out the microbunch, as seen in Fig. 13(c).

It is certainly possible that a more complicated misalignment existed during the experiment, including mismatch in $x, y$ spatial overlap, $\theta_{x}, \theta_{y}$ angular errors, and uncertainty where the $e$-beam and laser beam crossed inside the undulator (the model assumes in the center of the undulator). It is not practical to perform an exhaustive study of all the variations in misalignment. Furthermore, it would be of limited usefulness because the laser beam focus inside the undulator is not Gaussian and contains higher-order diffraction effects. Therefore, the primary purpose for suggesting the spatial offset is to demonstrate that such an effect has predictable consequences and is consistent with the spatial drift observed during the experiment.

\section{F. Data and model results versus phase delay}

All the data shown in Figs. 12 and 13 are at a chicane phase delay set for maximum acceleration. Figure 14 presents the energy spectrum data and model predictions as a function of the chicane phase delay assuming the same model parameters as used in Fig. 12. The only model parameter that was changed to generate the predictions given in Fig. 14 was the chicane phase delay.

We have arbitrarily assigned Fig. 14(a) to correspond to zero phase delay. This phase is also very close to the one used by the model in Fig. 12(a); hence, Fig. 14(a) represents the phase at maximum acceleration. Figure 14(b) is the corresponding energy-phase plot produced by the model for Fig. 14(a) and is essentially the same as the 


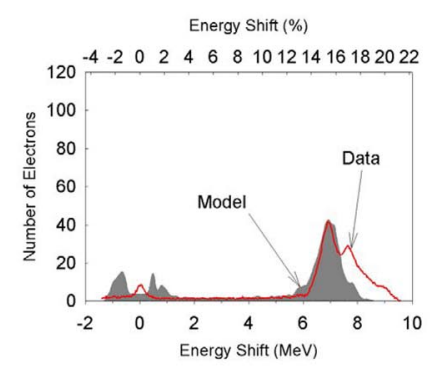

(a) Phase delay $=0^{\circ}$

Energy Shift (\%)

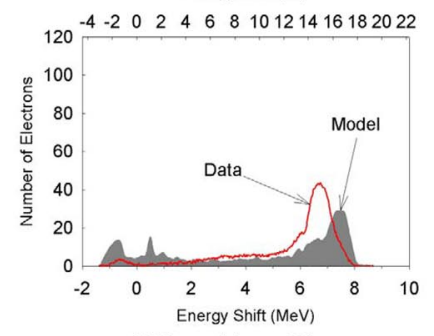

(c) Phase delay $=45^{\circ}$

Energy Shitt (\%)

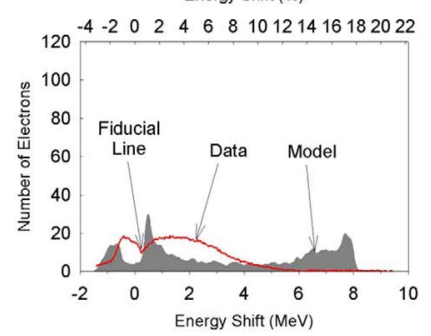

(e) Phase delay $=90^{\circ}$ (data), $65^{\circ}$ (model)

Energy Shift (\%)

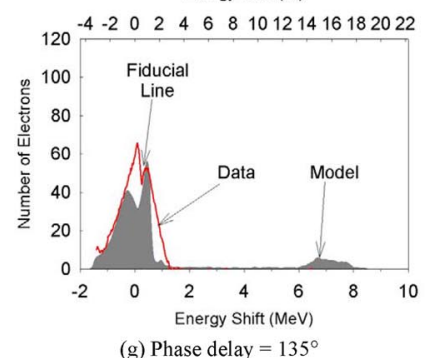

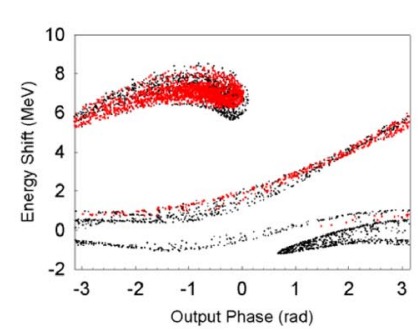

(b) Phase delay $=0^{\circ}$

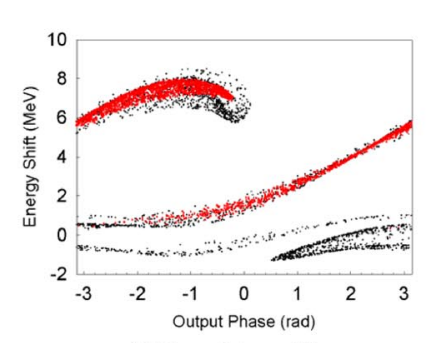

(d) Phase delay $=45^{\circ}$

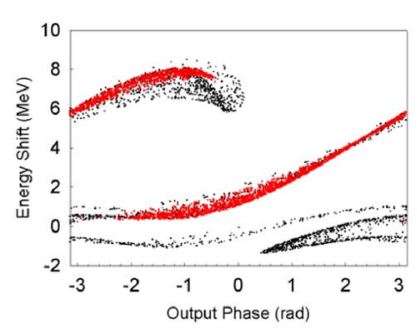

(f) Phase delay $=90^{\circ}$ (data), $65^{\circ}$ (model)

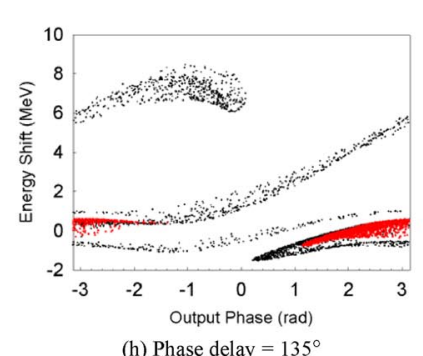

Energy Shift (\%)

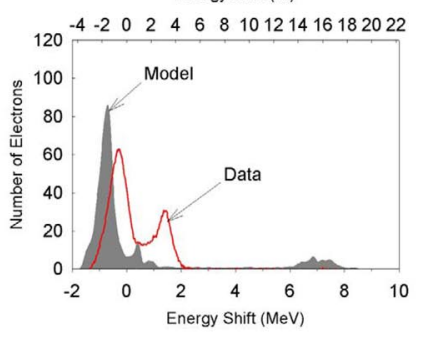

(i) Phase delay $=180^{\circ}$

Energy Shift (\%)

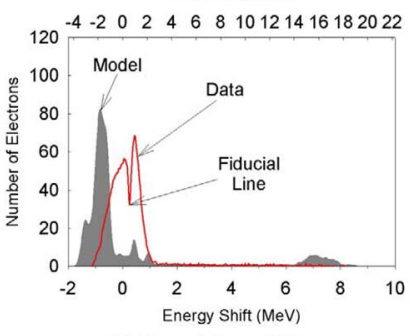

(k) Phase delay $=225^{\circ}$

Energy Shitt (\%)

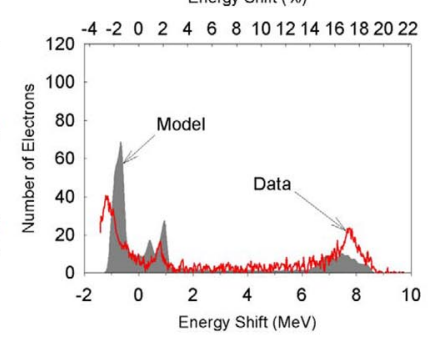

(m) Phase delay $=270^{\circ}$

Energy Shift (\%)

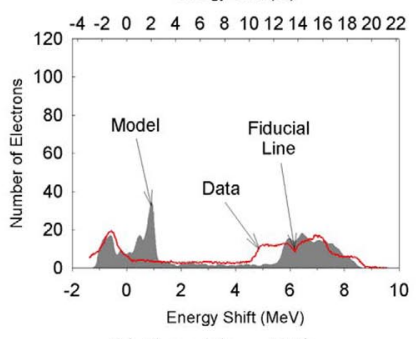

(o) Phase delay $=315^{\circ}$

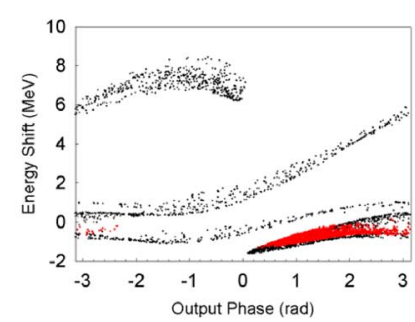

(j) Phase delay $=180^{\circ}$

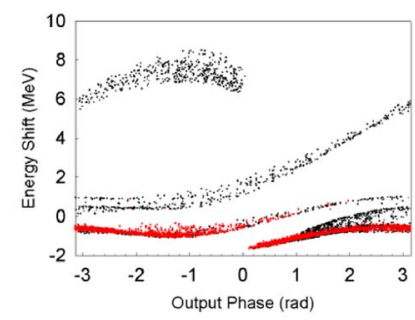

(1) Phase delay $=225^{\circ}$

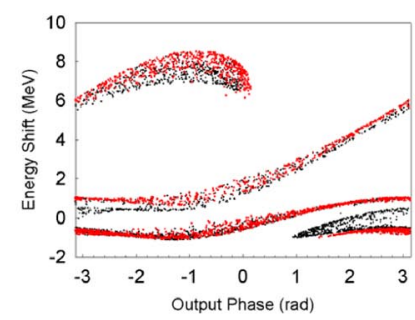

(n) Phase delay $=270^{\circ}$

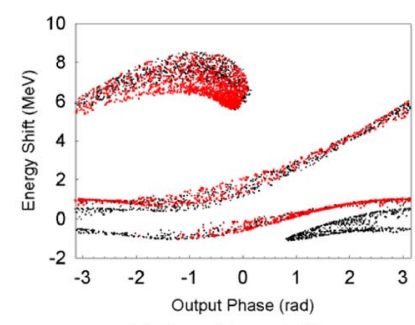

(p) Phase delay $=315^{\circ}$

FIG. 14. (Color) Comparison of data energy spectra with model prediction as a function of chicane phase delay. (a),(c),(e),(g),(i),(k),(m),(o) data is solid red curve; model is gray histogram. (b),(d),(f),(h),(j),(l),(n),(p) corresponding model energy-phase plots with initial microbunch electrons [see Fig. 11(b)] colored in red.

plot in Fig. 12(b) except for a change in axis orientation. (The orientation in Fig. 14 is the more commonly used one.) The microbunch electrons, which were labeled red in Fig. 11(b), are once again labeled red in Fig. 14(b).

As the phase is changed, these microbunch electrons move along the phase-space "pathway" through the three groups of untrapped electrons in the phase space mentioned earlier. This can be clearly seen in Figs. 14(d), 14(f), 14(h), and 14(j) where the microbunch electrons appear to slip out of the bucket and become dispersed along the middle phase-space pathway [Fig. 14(f)] until they become grouped again at the $180^{\circ}$-phase point [Fig. 14(j)] corresponding to maximum deceleration. As the phase increase continues, the process reverses itself and the microbunch electrons once again become smeared out in phase [Fig. 14(n)] until they begin to gather again in the acceleration bucket [Fig. 14(p)].

This movement of the microbunch electrons through phase space is manifested in the model energy spectra by the appearance of peaks of electrons in different portions of the spectrum. As can be seen, multiple peaks can occur depending on how the microbunch electrons are distributed among the various groups of untrapped electrons along the phase-space pathway.

The overall agreement between the data and model to first order is quite good. In particular, Figs. 14(a) and 


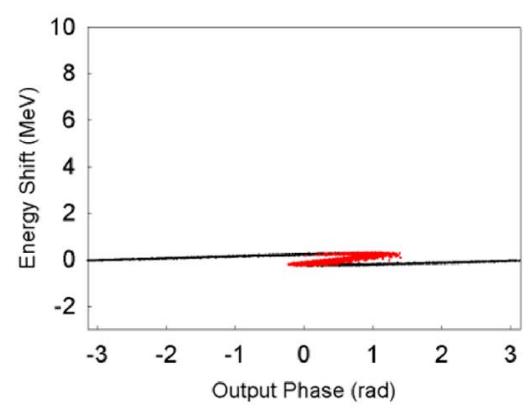

(a) At entrance to undulator

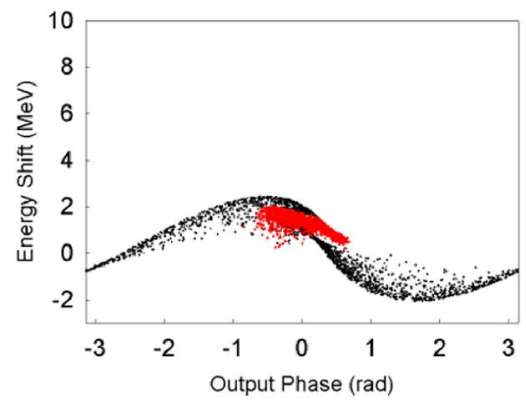

(c) $25 \%$ along undulator

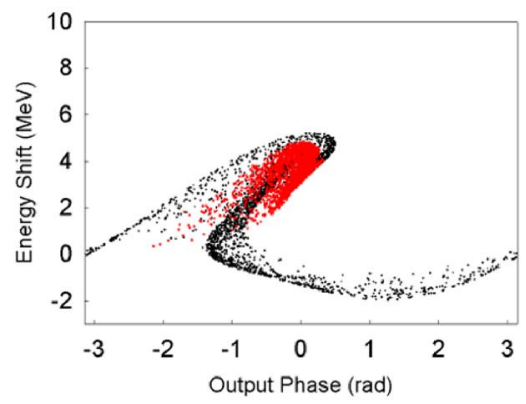

(e) $50 \%$ along undulator

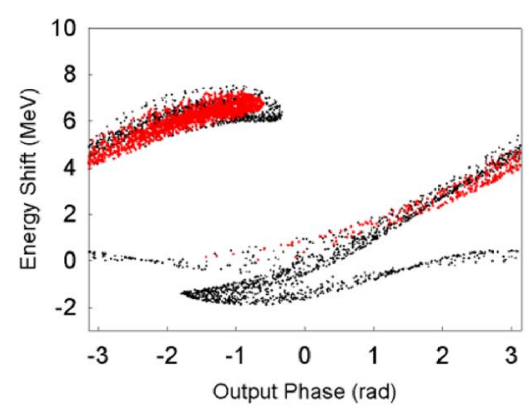

(g) $80 \%$ along undulator

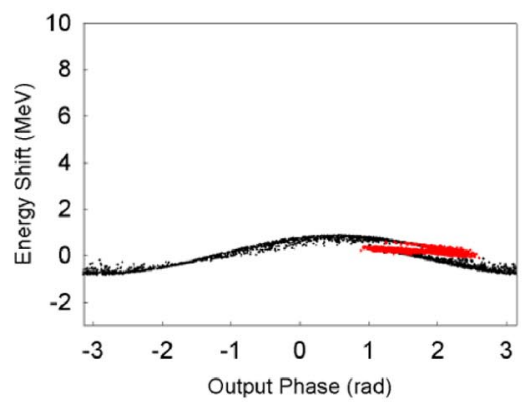

(b) $10 \%$ along undulator

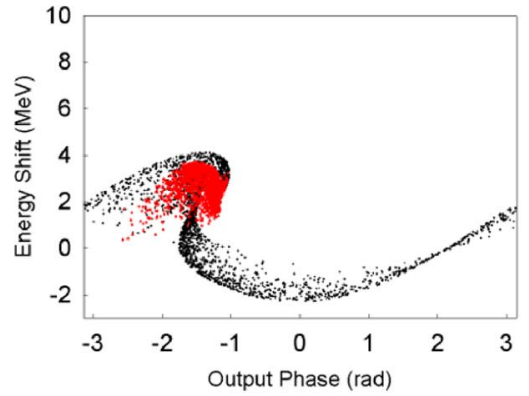

(d) $40 \%$ along undulator

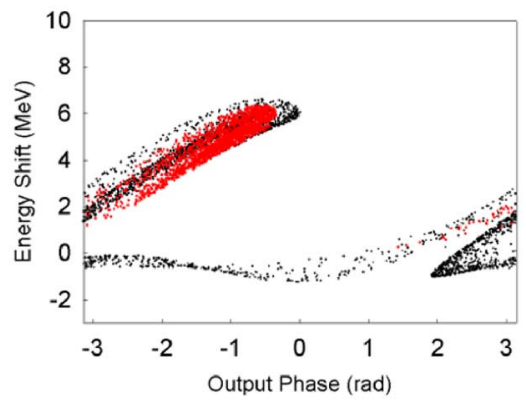

(f) $65 \%$ along undulator

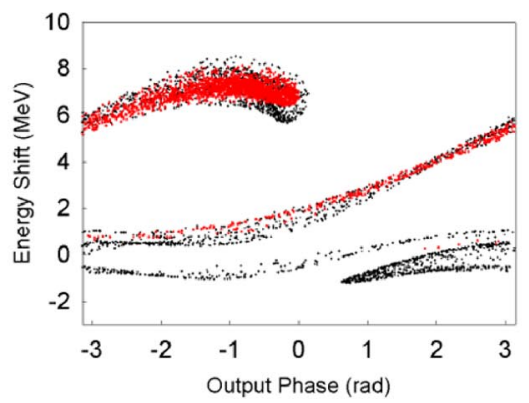

(h) Exit of undulator

FIG. 15. (Color) Model prediction for energy-phase plot at different positions along the tapered undulator of IFEL2 assuming the same model parameters used in Fig. 12. (a) Same plot as Fig. 11(b) with the initial microbunch electrons colored in red. (b)(f) Energy-phase plots corresponding to the percentage of distance along the undulator indicated. (h) Same plot as Fig. 12(b).

14(c) show very similar profiles for the accelerated electrons. Figures 14(i) and 14(m) display similar evidence of multiple peaks near zero energy shift. And, Figs. 14(m) and 14(o) also clearly show diminished peaks of accelerated electrons as predicted by the model. However, the diminished peak of accelerated electrons is not always observed in the data [see Figs. 14(g), 14(i), and 14(k)], which may be because the signal was too weak for the spectrometer camera.

There is less agreement between the data and model at a phase delay of $90^{\circ}$ [Fig. 14(e)]. Note, unlike the other plots in this figure where the experiment and the model phase delays are identical, in Fig. 14(e) the model phase delay is $65^{\circ}$ rather than $90^{\circ}$. This is the model delay that 
provided the best match with the data. If the model phase delay is set to $90^{\circ}$, then the microbunch electrons tend to shift too far towards the left in Fig. 14(f) within the central group of untrapped electrons along the middle phase pathway. This results in a large concentration of electrons at approximately $0.5 \mathrm{MeV}$ energy shift. This greatly accentuates the peak of electrons already seen at $0.5 \mathrm{MeV}$ in Fig. 14(e), resulting in gross disagreement with the data. The data has a much broader distribution in energy than the model and no evidence of any appreciable accelerated electrons. One possible explanation is that for this set of data the microbunch electrons are not as well formed and more spread out in phase.

We should emphasize that part of the residual differences between the data and model seen in Fig. 14 are due to naturally occurring variations in the delivered laser peak power and spatial overlap drift between the $e$-beam and laser beam. For example, as mentioned, the laser had $\mathrm{a} \pm 12 \%$ shot-to-shot variation. In addition, we intentionally operated near the minimum threshold needed to drive the tapered undulator in order to obtain the narrowest energy spread. Operation at this point also makes the shape of the spectrum particularly sensitive to changes in the laser peak power. Consequently, although the model parameters in Fig. 14 were kept constant except for the phase delay, in reality, the data conditions were probably not identical at each phase point. Hence, some residual differences between the data and model are to be expected.

Lastly, an example of a shot with low $e$-beam charge is shown in Fig. 14(m). This explains the higher degree of noise in the data. Note, the energy gain is still high and the energy spread of the accelerated electrons is still similar to the model prediction.

\section{FURTHER MODEL SIMULATIONS}

As shown in the previous figures, the energy-phase plots of the model greatly enhance our understanding of the electron dynamics occurring during this experiment. It is instructive to use the model to reveal how the hightrapping efficiency process occurs. Figure 15 gives the model energy-phase plots at different locations along the tapered undulator (IFEL2) for the conditions of Fig. 12. Figure 15(a) is at the entrance to IFEL2 and is the same as Fig. 11(b) with the axis compressed and orientation rotated. Figures $15(\mathrm{~b})-15(\mathrm{~g})$ represent the phase-space conditions at distances along the undulator of $10 \%-80 \%$, respectively. The final energy-phase plot at the exit of the undulator [Fig. 15(h)] is the same as Fig. 12(b).

Examining the figures, we see that neighboring electrons in phase on either side of the microbunch electrons are captured together with the microbunch electrons as they gain energy along the undulator. The origins of the three groups of untrapped electrons along the phase pathway are now clearly evident.

We also gain an understanding of how the net acceleration can exceed the amount of energy taper in the IFEL2 wiggler. (As shown earlier, the net energy gain of the trapped electrons is about $16 \%$ [Fig. 12(a)], whereas the wiggler energy taper is $12 \%$.) Since the trapped electrons are only just starting one synchrotron oscillation when they exit the wiggler [Fig. 15(h)], they end up near the top of the ponderomotive potential well bucket. This, together with initial injection at an energy slightly below resonance $(46.3 \mathrm{MeV})$ at the wiggler entrance, allows the final energy gain of the trapped electrons to significantly exceed the amount of energy taper of the wiggler.

\section{CONCLUSION}

The STELLA experiment demonstrated three important capabilities needed by a practical laser linac: (i) the ability to rephase microbunches produced by a laserdriven device with the optical field in another laser-driven device, i.e., staging; (ii) the ability to capture a large percentage of the microbunches in the ponderomotive potential well of the accelerating field, i.e., high-trapping efficiency; and (iii) the ability to accelerate the trapped microbunches while maintaining a narrow energy spread, i.e., monoenergetic. These accomplishments imply the possibly of using multiple acceleration stages to achieve high net energy gain with appreciable beam current and acceptable final energy spread.

Within the limitations of our ability to ascertain the values of all the experimental parameters, the model agrees well with the overall features of the data. At a finer level there are differences between the model and data. Improving the agreement at this level would require both more complete and accurate knowledge of the experimental conditions and upgrading the model to include simulation of a non-Gaussian laser beam with aberrations and utilization of 3D magnetic field measurements of all the devices.

IFELs were a convenient tool for studying the staging and trapping process. However, it is difficult to scale IFELs to high energies because the acceleration gradient scales as $1 / \gamma$ [see Eq. (16)] and eventually synchrotron losses become significant at high beam energies. Furthermore, the maximum practical acceleration gradient of IFELs is substantially smaller than possible with other laser acceleration techniques.

Fortunately, the STELLA approach of using a laserdriven modulator to produce microbunches followed by trapping and accelerating the microbunches in subsequent laser-driven devices can be applied to other schemes offering much better scaling potential. In particular, the STELLA experiment will be next investigating laser wakefield acceleration (LWFA) [1] driven by a $\mathrm{CO}_{2}$ laser beam at $10.6 \mu \mathrm{m}$. LWFA at $10.6 \mu \mathrm{m}$ has been analyzed 
[25] with gradients of the order of $1 \mathrm{GV} / \mathrm{m}$ predicted. It may have certain advantages over LWFA that uses shorter-wavelength lasers [26]. In addition, the ATF $\mathrm{CO}_{2}$ laser, which has been upgraded to produce terawatt peak powers [27], should produce laser pulses as short as $\approx 2$ ps. This pulse length is in an interesting pseudoresonant LWFA regime [25] that has also been referred to as forced LWFA [28]. Studying pseudoresonant LWFA is of interest because it may offer an easier way to control the highly nonlinear processes occurring in LWFA.

\section{ACKNOWLEDGMENTS}

This work was supported by the U.S. Department of Energy, Grants No. DE-FG03-98ER41061, No. DEAC02-98CH10886, and No. DE-FG03-92ER40695.

[1] W. P. Leemans and E. Esarey, in Advanced Accelerator Concepts: Eighth Workshop, edited by W. Lawson, C. Bellamy, and D. Brosius, AIP Conf. Proc. No. 472 (AIP, New York, 1999), p. 174.

[2] See http://www.bnl.gov/atf/Menu.html.

[3] I.V. Pogorelsky, J. Fischer, K. P. Kusche, M. Babzien, N. A. Kurnit, I. J. Bigio, R. F. Harrison, and T. Shimada, IEEE J. Quantum Electron., 31, 556 (1995).

[4] W. D. Kimura, G. H. Kim, R. D. Romea, L. C. Steinhauer, I.V. Pogorelsky, K. P. Kusche, R. C. Fernow, X. Wang, and Y. Liu, Phys. Rev. Lett. 74, 546 (1995).

[5] A. van Steenbergen, J. Gallardo, J. Sandweiss, and J. M. Fang, Phys. Rev. Lett. 77, 2690 (1996).

[6] L. P. Campbell, C.E. Dilley, S. C. Gottschalk, W. D. Kimura, L. C. Steinhauer, M. Babzien, I. Ben-Zvi, J.C. Gallardo, K. P. Kusche, I.V. Pogorelsky, J. Skaritka, A. van Steenbergen, V. Yakimenko, D. B. Cline, P. He, Y. Liu, and R. H. Pantell, IEEE Trans. Plasma Sci. 28, 1143 (2000).

[7] W. D. Kimura, A. van Steenbergen, M. Babzien, I. BenZvi, L. P. Campbell, C.E. Dilley, D. B. Cline, J.C. Gallardo, S. C. Gottschalk, P. He, K. P. Kusche, Y. Liu, R. H. Pantell, I.V. Pogorelsky, D. C. Quimby, J. Skaritka, L. C. Steinhauer, and V. Yakimenko, Phys. Rev. Lett. 86, 4041 (2001).

[8] W. D. Kimura, M. Babzien, I. Ben-Zvi, L. P. Campbell, D. B. Cline, C. E. Dilley, J. C. Gallardo, S. C. Gottschalk, K. P. Kusche, R. H. Pantell, I.V. Pogorelsky, D.C. Quimby, J. Skaritka, L.C. Steinhauer, V. Yakimenko, and F. Zhou, Phys. Rev. Lett. 92, 054801 (2004).

[9] R. B. Palmer, J. Appl. Phys. 43, 3014 (1972).

[10] E. D. Courant, C. Pellegrini, and W. Zakowicz, Phys. Rev. A 32, 2813 (1985).

[11] D. C. Quimby, S.C. Gottschalk, and W. D. Kimura, in Proceedings of the International Conference on Lasers 2001, Tucson, AZ, edited by V. J. Corcoran and T. A. Corcoran (STS, McLean, VA, 2002), p. 133.

[12] For more information, see www.stioptronics.com.
[13] W. D. Kimura, M. Babzien, I. Ben-Zvi, L. P. Campbell, D. B. Cline, C. E. Dilley, J. C. Gallardo, S. C. Gottschalk, K. P. Kusche, R. H. Pantell, I.V. Pogorelsky, D.C. Quimby, J. Skaritka, L.C. Steinhauer, V. Yakimenko, and F. Zhou, in Advanced Accelerator Concepts: Tenth Workshop, edited by C. E. Clayton and P. Muggli, AIP Conf. Proc. No. 647 (AIP, New York, 2002), p. 269.

[14] X. J. Wang, X. Qiu, and I. Ben-Zvi, Phys. Rev. E 54, R3121 (1996).

[15] F. Zhou, I. Ben-Zvi, M. Babzien, X.Y. Chang, A. Doyuran, R. Malone, X. J. Wang, and V. Yakimenko, Phys. Rev. ST Accel. Beams 5, 094203 (2002).

[16] V. Yakimenko, M. Babzien, I. Ben-Zvi, R. Malone, and X. J. Wang, Nucl. Instrum. Methods Phys. Res., Sect. A 483, 277 (2002).

[17] C. A. Brau, Free-Electron Lasers (Academic, New York, 1990), p. 156.

[18] N. M. Kroll, P. L. Morton, and M. N. Rosenbluth, IEEE J. Quantum Electron. 17, 1436 (1981).

[19] D. C. Quimby, C. G. Parazzoli, and D. J. Pistoresi, Nucl. Instrum. Methods Phys. Res., Sect. A 318, 628 (1992).

[20] I.V. Pogorelsky, I. Ben-Zvi, T. Hirose, S. Kashiwagi, V. Yakimenko, K. Kusche, P. Siddons, J. Skaritka, T. Kumita, A. Tsunemi, T. Omori, J. Urakawa, M. Washio, K. Yokoya, T. Okugi, Y. Liu, P. He, and D. Cline, Phys. Rev. ST Accel. Beams 3, 090702 (2000).

[21] I. Pogorelsky, I. Ben-Zvi, W. D. Kimura, N. A. Kurnit, and F. Kannari, Infrared Phys. Technol. 36, 341 (1995).

[22] W. D. Kimura, L. P. Campbell, C.E. Dilley, S.C. Gottschalk, D.C. Quimby, A. van Steenbergen, M. Babzien, I. Ben-Zvi, J.C. Gallardo, K. P. Kusche, I.V. Pogorelsky, J. Skaritka, V. Yakimenko, D. B. Cline, P. He, Y. Liu, L. C. Steinhauer, and R. H. Pantell, Phys. Rev. ST Accel. Beams 4, 101301 (2001).

[23] F. Zhou, D. B. Cline, and W. D. Kimura, Phys. Rev. ST Accel. Beams 6, 054201 (2003).

[24] W. D. Kimura, M. Babzien, I. Ben-Zvi, L. C. Campbell, D. B. Cline, C. E. Dilley, J. C. Gallardo, S. C. Gottschalk, K. P. Kusche, R. H. Pantell, I.V. Pogorelsky, D.C. Quimby, J. Skaritka, L.C. Steinhauer, V. Yakimenko, and F. Zhou, in Proceedings of the 2004 Advanced Accelerator Concepts Workshop, Stony Brook, NY (to be published).

[25] N.E. Andreev, S.V. Kuznetsov, A. A. Pogosova, L.C. Steinhauer, and W. D. Kimura, Phys. Rev. ST Accel. Beams 6, 041301 (2003).

[26] I.V. Pogorelsky, Nucl. Instrum. Methods Phys. Res., Sect. A 410, 524 (1998).

[27] I.V. Pogorelsky, I. Meshkovsky, A. Dublov, I. Pavlishin, Yu. A. Boloshin, G. B. Deineko, and A. Tsunemi, in Proceedings of the International Conference on Lasers 1998, Tucson, AZ, edited by V. J. Cocoran and T. A. Goldman (STS, McLean, VA, 1999), p. 911.

[28] Z. Najmudin, K. Krushelnick, E. L. Clark, S. P. D. Mangles, B. Walton, A. E. Dangor, S. Fritzler, V. Malka, E. Lefebvre, D. Gordon, F. S. Tsung, and C. Joshi, Phys. Plasmas 10, 2071 (2003). 